Csaba Földes (Veszprém)

\title{
Grammatische Zweisamkeit. Morphosyntax im Sprachen- und Kulturenkontakt
}

\begin{abstract}
Der Aufsatz diskutiert grammatische Aspekte von authentischen Sprachgebrauchsstrukturen in einem komplexen Kontakt- und Integrationsraum von mehreren Sprachen und Kulturen. Als empirisches Illustrationsmaterial dient ein umfangreiches kontaktlinguistisches Feldforschungsprojekt im ungarndeutschen Ort Hajosch/Hajós (Komitat Batsch-Kleinkumanien/Bács-Kiskun). Anhand von dort ermittelten Sprechprodukten zwei- bzw. mehrsprachiger Sprecher werden vielgestaltige sprachlich-kommunikative Kontakt-, Konvergenz- und Interaktionsphänomene grammatischer Natur identifiziert. Ihre Analyse ergab, dass die exemplarisch untersuchte Diskursgemeinschaft beim Umgang mit morphosyntaktischen Phänomenen zahlreiche und vor allem mannigfaltige Formen von Hybridität hervorbringt. Die erschlossenen Phänomenklassen und -typen scheinen für transkulturelle Zusammenhänge generell verallgemeinerbar zu sein.
\end{abstract}

This article discusses grammatical aspects of authentic linguistic usage structures in an area of complex contact and integration involving several languages and cultures. Empirical illustrative material is provided by an extensive field research project on language contact in the Hungarian-German village of Hajosch/Hajós (BácsKiskun county). A great variety of grammatical phenomena relating to linguistic and communicative contact, convergence and interaction are identified on the basis of the speech of bi- and/or multilingual speakers recorded there. The analysis of these phenomena reveals that the discourse community studied here produces numerous and above all varied forms of hybridity in the process of handling morphosyntactic phenomena. The classes and types of phenomena identified here seem to occur in transcultural contexts generally.

\section{Forschungshorizont und Zielsetzung}

Der vorliegende Beitrag setzt sich mit einer 'Kultur von Mehrsprachigkeit' in einer interbzw. transkulturellen ${ }^{1}$ „Fugen-Position“ auseinander und fußt auf einem größeren kontaktlinguistischen Feldforschungsprojekt (vgl. FÖLDES 1996, 2005). Es konzentriert sich auf aktuelle ungarndeutsche Sprachgebrauchsstrukturen in einem komplexen Kontakt-, Konvergenz- und Integrationsraum von mehreren Sprach(varietät)en sowie Kulturen und möchte dadurch zur Modellierung bi- bzw. multilingualen Diskursverhaltens auf verschiedenen Ebenen beitragen.

Die empirische Datenbasis stammt aus dem ungarndeutschen Ort Hajosch/Hajós in der nördlichen Batschka, im Komitat Batsch-Kleinkumanien/Bács-Kiskun. Das Projekt geht phänomenorientiert und problembezogen vor und soll differenziert erschließen, wie sich der Kontaktdruck des Ungarischen als Modell-, Bezugs- und Überdachungssprache in mündlichen Diskursen innerhalb der Vernakularsprache der deutschen MinderheitenGemeinschaft auf der synchronen Ebene äußert. ${ }^{2}$ Im Einzelnen geht es also darum, am Material von authentischen oralen Sprechprodukten zwei- bzw. mehrsprachiger Personen in einem multi- bzw. transkulturellen sozialen Kontext vielgestaltige (evidente und latente) sprachlich-kommunikative Kontakt- sowie Interaktionsphänomene zu erfassen, zu typisieren und zu explizieren.

\footnotetext{
${ }^{1}$ Zur Konzeptualisierung dieser Begrifflichkeit siehe FöLDES (2003: 53 f.).

${ }^{2}$ Forschungsdesign, Grundkonzept und Hauptlinien wurden in FöLDES (1996, 2005) detailliert ausgeführt.
} 
Zentrale Begriffe meines terminologischen Apparats bilden 'kommunikativer Synkretismus' und 'sprachliche Hybridität'. Mit dem Terminus 'Synkretismus' - als Bemühung um Harmonisierung unterschiedlicher Systeme - (vgl. BERNER 1982) wurde in der Linguistik bisher kaum operiert; geläufig ist der Begriff lediglich in einem ganz anderen Sinne als 'formaler Zusammenfall verschiedener, ursprünglich getrennter grammatischer Funktionen', was besonders anhand des Kasussystems verschiedener Sprachen als sog. Mischkasus eine Rolle spielt (vgl. CARSTAIRS-MCCARTHY 1994). In Bezug auf die 'Hybridisierung' gehen viele Definitionsversuche z.T. auf BACHTINs kultursemiotisches Konzept (1979: 244) zurück: „Vermischung zweier sozialer Sprachen innerhalb einer einzigen Äußerung“. Indes besteht bei der Verwendung des in den Fachdiskursen mittlerweile etablierten „poststrukturalistischen“ Begriffs 'Hybridität' (und mit ihm verbundener Termini) eine Schwierigkeit in der unterschiedlichen Vorstellung hinsichtlich Extension und Abgrenzung von ähnlichen oder benachbarten Konzepten, insbesondere weil der Begriff aus unterschiedlichen Argumentationszusammenhängen bzw. Wissenschaftskontexten stammt und sich auf verschiedene Objektbereiche bezieht. Hinzu kommt, dass das Lexem 'hybrid' im Deutschen vielfach einen schweren Status hat. ACKERMANN (2004: 140) weist sogar darauf hin, dass sich dieser Begriff einer eindeutigen Verortung verweigert und häufig ,im Metaphorischen“" verbleibt, indem er „Transformationen gegen Kontinuität und Mehr- gegen Eindeutigkeit" setzt. ${ }^{3}$ In meinem Begriffsapparat bezieht sich 'Synkretismus' eher auf die Sprechhandlung, während 'Hybridisierung' im Hinblick auf die sprachlichen (sprachsystematischen) Prozesse verwendet wird.

In diesem Bezugsrahmen behandelt der vorliegende Beitrag grammatische Phänomene an der Schnittstelle zwischen zwei Sprachsystemen, mit besonderer Berücksichtigung des Konstrukts 'Transferenzen' (zur verwendeten Sprachenkontakt-Terminologie vgl. FöLDES 1996: 12 ff., 2005: 63 ff.). Dabei will der Aufsatz ermitteln, wie morphosyntaktische Phänomene in gemischtsprachigen Diskursen unter Bedingungen einer transkulturellen Mehrsprachigkeit auftreten, indem ihre Realisationsstrukturen, -typen und -klassen erschlossen sowie ihr Funktionieren hinterfragt werden. Letzten Endes soll anhand der Auseinandersetzung mit einer vitalen und hochkomplexen Kontaktsituation von Sprachen bzw. Varietäten der sprachkommunikative Umgang mit Grammatik aus der Sicht des Kulturphänomens 'deutsche Sprache' im Kräftefeld von typologischer Tradition und sukzessiver Innovation beschrieben und gleichzeitig der Kontaktprozess modelliert werden. In diesem Kontext steht die empirisch fassbare Variationsbreite von arealen Sprachenkontaktbzw. -interaktionserscheinungen - in ihrer Systematik und interaktiven Dynamik - im Fokus. Konzeptionell betrachtet, geht es um die generelle theoretische und methodologische Frage, wie der Makrokosmos bilingualer und transkultureller sprachkommunikativer Handlungssysteme angemessen erfasst werden kann.

\section{Die Materialgrundlage}

Im Falle des gegebenen Forschungsobjektes - nämlich der Sprache bzw. des Sprachgebrauchs einer deutsch(sprachig)en Minderheiten-Gemeinschaft in Ungarn - ist zu betonen, dass der Realitätsbereich 'Deutsch als Minderheitensprache' naturgemäß in einem völlig anders gearteten soziokulturellen Referenzrahmen existiert als die binnendeutsche Standardvarietät, aber auch als die binnendeutschen Dialekte der Gegenwart. Vor allem durchgreifende Zweibzw. Mehrsprachigkeit sowie eine enge und intensive Kontaktstellung zum Ungarischen (und

\footnotetext{
${ }^{3}$ Zur Hybriditäts-Debatte siehe BHABHA (2000: 5, 7 etc.).
} 
z.T. zu anderen Minderheitensprachen) sind dabei konfigurationskonstitutiv (siehe ausführlicher FöLDES 2005: 44 ff.).

Bei den Ungarndeutschen haben - auch in Hajosch - die lebensweltliche Mehrsprachigkeit und die Diglossie in den letzten Jahrzehnten in quantitativer wie auch in qualitativer Hinsicht einen grundlegenden Transformationsprozess durchlaufen: Sowohl die sprachlichen Formen als auch die Diskursrealisierungen sind gleichzeitig auf der Mikro- und Makroebene durch eine außerordentlich hohe Dynamik gekennzeichnet. Mitunter zeigen sich sogar Ansätze von Fluktuation sowie u.U. eine zunehmende Labilität von Sprach- bzw.

Kommunikationsstrukturen. Folglich ist Okkasionalität ein zentrales Merkmal ungarndeutscher Redeweise. Inzwischen findet Ungarisch (als H-Varietät) eigentlich in allen Primär- und Sekundärdomänen häufig, vorwiegend oder ausschließlich Verwendung, während der ungarndeutsche Ortsdialekt seine meisten Funktionen verloren hat und sich (als L-Varietät) auf die Primärdomäne 'Familie' zurückgezogen hat, wobei er selbst in diesem „Refugium“ immer häufiger dem Ungarischen weicht. ${ }^{4}$ Andererseits deutet sich stellenweise eine gewisse Sprachvarietäten-Umstellung an, indem die deutsche Standardsprache zuweilen auch in Domänen eingesetzt wird, die bislang weitgehend durch den Dialekt oder durch die ungarische Standardvarietät geprägt waren.

Die unübersehbare kommunikative Expansion des Ungarischen ergibt stufenweise neuere Kontakt- bzw. Mischformen/-konfigurationen auf den verschiedenen Ebenen, aber ganz vornehmlich in der Lexik, Phraseologie und Pragmatik. Die Frage der Sprachentrennung ergibt sich bei Mitgliedern bi- oder multilingualer Diskursgemeinschaften - zumindest im sog. bilingualen Diskurs- bzw. Interaktionsmodus (vgl. FöLDES 2005: 65 ff.) - nicht oder zumindest ganz anders als bei einsprachigen Menschen und Gemeinschaften. Unter identisch mehrsprachigen Personen ist eine hybride Redeweise die natürliche und übliche Varietät und sozialpsychologisch gesehen hat die hybridisierte ${ }^{5}$ Sondervarietät eine nicht unwesentliche Funktion als mögliches Symbol regionaler Loyalität bzw. Identität. In den verschiedenen Kommunikationszusammenhängen wird im Wesentlichen auf drei sprachliche Kodes und ihre subtilen Übergangs- bzw. Mischformen zurückgegriffen, und zwar auf den jeweiligen ungarndeutschen Ortsdialekt (in Hajosch: Schwäbisch), auf die ungarische Standardsprache und auf die deutsche Standardvarietät. Die Hauptkomponenten des Kontakts bilden also ein (ungarndeutscher) Dialekt und eine exogene - die ungarische - Standardsprache, neben weiteren anderen. Das heißt, die Situation ist durch eine Zwei- bzw. Mehrsprachigkeit strukturell „unähnlicher“ Sprachen mit „ungleichwertigem“ Status und Prestige geprägt. Dies führt zu einem asymmetrischen Charakter des Sprachenkontaktes. Die Konstellation könnte man in Ermangelung eines etablierten Terminus vielleicht „bilinguale Dialekt-StandardDiglossie ${ }^{\text {c6 }}$ nennen. Den vor diesem Hintergrund in der Ingroup-Kommunikation verwendeten Kode bezeichne ich als „Kontaktdeutsch“(vgl. FöLDES 2005: 29 ff.).

\section{Grammatische Phänomene an der Schnittstelle von zwei Sprachen}

\footnotetext{
${ }^{4}$ Das heißt aber nicht automatisch, dass für diese Sprecher die ungarndeutsche ethnisch-sprachlich-kulturelle Herkunft keine Rolle mehr spielt. Denn viele haben in den letzten Jahrzehnten eine unilinguale, jedoch bi- bzw. transkulturelle Primärsozialisation durchlaufen: Ungarisch dient als Familien- und Umgebungssprache, aber im Alltag haben ungarndeutsche Sitten, Bräuche, Essgewohnheiten etc. einen festen Platz. Sprache ist mithin ein zentrales, aber keineswegs das alleinige konstitutive Merkmal einer ethnisch-nationalen Gruppe.

${ }^{5}$ Zum Konstrukt 'Hybridität' vgl. die Ausführungen von FöLDES (2005: 16).

${ }^{6}$ Oder 'Triglossie', weil neben den beiden Hauptakteuren der Kontaktsituation (der ungarndeutsche Ortsdialekt und die ungarische Standardsprache, die also zwei Sprachsystemen angehören) eigentlich auch die deutsche Standardvarietät eine nicht unwichtige Rolle spielt.
} 
3.1. In seinem kürzlich herausgebrachten ,donauschwäbischen“ Dialektkompendium meint GERESCHER (o.J.: 5) radikal: „Grammatik - tes is was, wumr trhom netkhat hen, weil mrs net gapraucht hot" (Standarddeutsch: Grammatik - das ist etwas, was wir daheim nicht hatten, weil wir es nicht gebraucht haben). Es bedarf wohl keines erläuternden Kommentars, dass diese absurde Feststellung mit der tatsächlichen Sprach- und Kommunikationsrealität nichts zu tun hat. Zur Vorstellung der aktuellen Verhältnisse hinsichtlich der Morphosyntax in einem Spagat zwischen (zwei) Sprachen und Kulturen sollen nun die im Belegkorpus ermittelten Realisierungsformen von Hybridität in der Grammatik genauer betrachtet und systematisch ausgewertet werden (vgl. detaillierter FöLDES 2005: 149 ff.).

Für die Forschungsliteratur bleiben diese Fragestellungen anscheinend nach wie vor in wichtigen Punkten (von den Kreolsprachen eventuell abgesehen; vgl. WINFORD 2003: 304 ff.) eine terra incognita.

MÜLLER behauptete in seiner erstmals 1861 erschienenen Schrift, dass „Sprachen in ihrem Vokabular zwar gemischt sein können, aber in ihrer Grammatik nie gemischt werden können“" (1965: 79). Diese Ansicht wurde von den nachfolgenden Forschergenerationen nahezu zu einem Dogma erhoben. So glaubte SCHAPPELLE Anfang des 20. Jahrhunderts, in der Syntax der deutschen Sprache von „,deutschbrasilianischen“ Kolonisten noch keine portugiesischen Einflüsse nachweisen zu können (1917: 42). Noch heute vertreten nicht wenige Linguisten den Standpunkt, dass zwischensprachliche morphosyntaktische Beeinflussungen praktisch nicht möglich seien. So bestreitet beispielsweise FILIPOVIć (1986: 185) für sog. direkte Sprachenkontakte die Möglichkeit einer syntaktischen Transferenz. ${ }^{7}$ Der entsprechende Wörterbuchartikel in einer linguistischen Enzyklopädie schränkt die Möglichkeit grammatischer Transferenzen auf die genetische Sprachverwandtschaft ein: Die Entlehnung von Wörtern mit grammatischen Funktionen und von formbildenden Affixen sei nur ,,möglich bei engen sprachlichen Kontakten verwandter Sprachen“ (IVANOV 1990: 237). Um weitere spezielle Beispiele aus der Sprache deutscher Minderheiten im östlichen Europa und in der GUS zu zitieren, verweise ich etwa auf den Standpunkt von BARBA (1982: 181): Sie habe bei den Rumäniendeutschen des Banats im morphosyntaktischen Bestand ,keine merklichen Spuren“ der Kontaktsprachen Ungarisch und Rumänisch registrieren können. Analog meint STEPANOVA (1983: 198 f.) anhand ihrer Forschungen an russlanddeutschem Sprachmaterial, die Syntax sei derart stabil, dass sie selbst in Dialekten, die in anderssprachiger Umwelt existieren, keine zwischensprachlichen Beeinflussungen erführe. Auch JEDIG konnte trotz eines ,sorgfältige[n] Vergleich[s] der Wortfolge des Satzes im Russischen und des Satzes der niederdeutschen Mundart" bei Russlanddeutschen keinen Einfluss des Russischen entdecken (BEREND/JEDIG 1991: 182). ${ }^{8}$ In Bezug auf die Kontaktsprachen Portugiesisch und Deutsch bescheinigte ZIEGLER (1996: 62) im Falle der „Deutschbrasilianer“: „Interferenzen formbildender Hilfsmorpheme“ waren ,für den deutsch-portugiesischen Sprachkontakt [...] nicht zu belegen gewesen. Ebenso sind wortbildende Hilfsmorpheme, wie Prä- und Suffixe, als Interferenzen der morphosyntaktischen Ebene innerhalb des deutsch-portugiesischen Sprachkontaktes nicht nachzuweisen.“

Selbst solche zeitgenössischen Publikationen, die mit einem ungarndeutschen Korpus arbeiten und als durchaus seriös einzustufen sind, belegen für die Bereiche der Syntax kaum Wirkungen von Sprachenkontakten. So resümiert WILD (1994: 98) die Resultate ihrer

\footnotetext{
${ }^{7}$ Es ist ferner auf SAPIR (1921: 203) und hinsichtlich der zeitgenössischen Forschung auf BÁTORI (1980: 134) hinzuweisen, die beide die Möglichkeit einer substanziellen morphologischen Beeinflussung bestreiten.

${ }^{8}$ Damit im Einklang stehend, konstatiert KLASSEN (1994: 71) für die von ihm untersuchten Sprachvarietäten der Russlanddeutschen: „Grammatische Einflüsse konnten ebenfalls nicht festgestellt werden“.
} 
Forschungen damit, dass ein Einfluss der ungarischen Sprache auf die Stellung der Satzelemente nicht mit Sicherheit nachgewiesen werden könne.

Das von mir ausgewertete Material deckt sich jedoch ganz und gar nicht mit jenen Aussagen, welche die Möglichkeit von morphosyntaktischen Transferenzen ausschließen bzw. welche bei der Analyse ihres Untersuchungsmaterials keine derartigen Transferenzen erschlossen haben. Um es gleich vorwegzunehmen: Ich konnte eine Reihe relevanter morphologischer und syntaktischer Kontakterscheinungen und kontakt-induzierter

Grammatikalisierungsphänomene ${ }^{9}$ belegen (die allerdings wirklich gegenstandskonform nur in einem diachronen Kontext anzugehen wären). ${ }^{10}$ Diese morphosyntaktischen Spezifika und Typika gehen in mancher Hinsicht wesentlich über das hinaus, was an anderen Sprachenpaaren unter anderen sprachsoziologischen Konfigurationen beschrieben worden ist oder sie zeigen dazu nur teilweise Parallelen. HufEISEN (1995: 245) hat z.B. die englischen Transferenz-Einwirkungen im Deutsch von deutschsprachigen Einwanderern in Kanada analysiert und gelangte zu der Erkenntnis: „Im Bereich der Morphologie gibt es zwei Übernahmemechanismen: Englische Verben werden nach deutschen Regulierungen konjugiert, deutsche Nomen werden nach englischer Morphologie dekliniert.“ Ersteres Phänomen - allerdings eher in den Vergangenheitsformen - war analog auch in meinem Beobachtungsmaterial zahlreich vertreten (vgl. Nr. 22), doch im Präsens wurde gewöhnlich das ungarische Infinitivsuffix behalten (vgl. Nr. 62). Die von HUFEISEN genannte zweite Besonderheit (1995: 247) kommt mir eher untypisch vor, zumal bei ihr je nach Zuordnung lediglich drei oder vier Items auftreten, die aber wenig überzeugend erscheinen, wie etwa Beginner ('Anfänger') oder Konsumer ('Konsument'). Ich sehe bei diesen Belegen keine englischen Pluralendungen an deutschen Substantiven und halte daher diese Beispiele für einfache lexikalische Transferenzen aus dem Englischen.

\subsection{In meinem Datenmaterial liegt dagegen eine Vielfalt aussagekräftigerer Fälle} grammatischer ,Zweisamkeit“" von zwei Sprachsystemen vor. So findet man im Beleg Nr. (1) in einem lexikalisch relativ homogenen - einsprachig deutschen - Satz ein als morphologischer Transfer aus dem Ungarischen explizierbares Phänomen: ${ }^{11}$

\footnotetext{
${ }^{9} \mathrm{Zu}$ den Grundannahmen, Argumentationsweisen und Definitionen der - bislang vorwiegend sprachtypologisch-universalistisch orientierten - Grammatikalisierungsforschung vgl. z.B. DIEWALD (1997) und HOPPER/TRAUGOTT (2000). Eine integrative und auf eine breitere empirische Basis rekurrierende Grammatikalisierungstheorie wird z.B. von GIRNTH (2000) angestrebt. Grammatikalisierungsphänomene besitzen in meinem transkulturellen Kontext auch deswegen Relevanz, weil von Sprache zu Sprache unterschiedlich ist, welche konzeptuellen Domänen bevorzugt mit grammatischen Formkategorien ausgedrückt werden.

${ }^{10}$ Das hängt wohl mit der fortgeschrittenen Stufe der Sprachumstellung in der untersuchten Diskursgemeinschaft zusammen, wenn nämlich die Sprecher das Ungarische (als eigentliche Zweitsprache) bereits besser beherrschen als das Deutsche (d.h. ihre eigentliche Erstsprache); vgl. die „IV. Phase“ im Modell von MELIKA (1994: 298 ff.). ${ }^{11} \mathrm{Zu}$ den Einzelheiten der verwendeten „Grobtranskription“"vgl. FöLDES (2005). Ich bediene mich in all den Fällen, wenn die Phonem-Graphem-Beziehungen des binnendeutschen Standards auch im dargestellten Dialekt gelten, schlicht und einfach des deutschen Schriftalphabets. Bei Abweichungen von diesen Korrespondenzen wird mit folgenden ergänzenden Zeichen operiert: Zur Kennzeichnung derjenigen langen Vokale des Dialekts, die im binnendeutschen Standard nicht lang sind wie auch zur Markierung von langen Konsonanten dient ein nachgestellter Doppelpunkt. Das Zeichen 'á' steht für einen (wohl aus dem Ungarischen stammenden) Laut, der unter artikulatorischen Aspekten ein kurzer gerundeter Hinterzungenvokal mit tiefer Zungenlage und weit jedoch nicht mit weitest - geöffnetem Kieferwinkel ist und unter akustischem Aspekt eine dunkle Klangfarbe besitzt. In Zweifelsfällen verschiedener Art habe ich stets (der besseren Lesbarkeit halber) standardnähere Schreibungen bzw. der geschriebenen Sprache näher stehende Formen bevorzugt. In meiner Notation erscheinen die Elemente ungarischer Provenienz bei allen Belegen gemäß der ungarischen Orthographie; typographisch werden sie - zur prägnanteren Kennzeichnung und Hervorhebung - durch Fettdruck markiert.
} 
(1) Käi 's nu: a Mischtháufara! $!^{12}$ (Standarddeutsch, im Weiteren SD: Gehei [= Wirf] es nur auf den Misthaufen!)

Diese Übernahme von Flexionsmorphemen erfolgt normalerweise sehr selten, zumal sie in hohem Maße integrierte und strukturierte Elemente der Sprache sind. Die Erfahrungen legen nahe: Je komplexere grammatische Funktionen diese erfüllen, desto geringer ist die Wahrscheinlichkeit ihres Transfers. Im Beleg Nr. (1) kann die Verwendung des ungarischen Artikels - falls dieses 'a' überhaupt als ungarischer Artikel und nicht als ein simpler Häsitationslaut eingestuft wird - u.U. auch dahingehend interpretiert werden, dass die Sprecherin das Substantiv Mischthaufa (SD: 'Misthaufen') als ungarisches Element behandelt. Sollte es so sein, hat man es hier nicht mit einer grammatischen Transferenz (als sprachliche Hybridität), sondern mit einer Kode-Umschaltung mitten im Satz (als kommunikativer Synkretismus) zu tun.

Im Datenkorpus sind mithin zahlreiche Belege für Mischungen im grammatischen Bau enthalten. Es gibt also Strukturen, bei denen die Bezugsgrammatik u.U. sowohl deutsch als auch ungarisch ist, z.B.:

(2) Käm:id a:u wentscházni! (SD: Kommt [eigentlich: Kommen Sie] auch [etwas] wünschen!) ${ }^{13}$

Der obige Befund für Sprachenmischung kann einerseits so ausgelegt werden, dass ein Übergang von dem einen zum anderen Sprachsystem bei der Infinitivendung erst im gegebenen Redemoment auftritt (entweder als 'Kode-Umschaltung' oder als 'grammatische Transferenz' interpretierbar). Man könnte andererseits auch annehmen, dass der Sprecher bereits das ganze Verb als ungarisch (bzw. hungarisiert) betrachtete. In diesem Fall wäre eher von einem Wort(rück-)transfer zu sprechen.

Noch überzeugender ist der folgende Fall von morphosyntaktischer Doppelmarkierung:

(3) Schit:'s miar ans Gläsliba $!^{14}$ (SD: Schütte [= Gieße] es mir ins Gläslein!)

Da ja die Kontraktion aus Präposition (in) und Artikel (das) noch deutsch ist, erfolgt hier die Hybridiserung zweifellos durch das ungarische Illativsuffix am Wortende, was zu einer hybriden Morphemstruktur führt. Ein möglicher Grund für diesen Transfer der ungarischen Morphologie dürfte in der sprachökonomischen ${ }^{15}$ Leistung des Ungarischen in diesem Bereich liegen, nämlich darin, dass raumbezogene Richtungsangaben im Ungarischen durch die jeweiligen Illativsuffixe rein morphologisch realisiert werden, während man sie im Deutschen etwas aufwändiger durch Präfixe mit den davon abhängenden (und indirekt vom Verb bestimmten) Kasus in den Artikeln und Kernsubstantivendungen der Nominalgruppe und somit morphosyntaktisch - ausdrückt. Überdies zeigt dieser Beleg eine eigentümliche Dualität. Die grammatischen Relationen kommen doppelt (also in beiden Sprachen) und zudem mit kategorial unterschiedlichen Beziehungsmitteln zum Ausdruck: im Deutschen analytisch und im Ungarischen synthetisch. Eine weitere Auffälligkeit ist dabei die Frage der Vokalharmonie. Durch Nachfragen bei den Informant(inn)en und aufgrund weiterer analoger

\footnotetext{
${ }^{12}$ Das vorangegangene $\boldsymbol{a}$ ist ein bestimmter Artikel des Ungarischen und -ra ist ein ungarisches Sublativsuffix (Ortsbestimmungssuffix der Oberfläche auf die Frage ,wohin?').

${ }^{13}$ Praktisch schon ganz ungarisch wirken hybride Sätze wie $\boldsymbol{A}$ Csöpi paisázik (SD: Der Csöpi [= Hundename] beißt) oder Schlafáztam egy kicsit (SD: Ich habe etwas geschlafen).

${ }^{14}$ Die Endung - $\boldsymbol{b a}$ ist ein ungarisches Illativsuffix.

${ }^{15}$ Zur Problematik 'Ökonomie in der Sprache' vgl. ausführlicher z.B. RONNEBERGER-SIBOLD (1980) und besonders WURZEL (2001).
} 
Beispiele wurde klar, dass in diesem Beleg im Prinzip auch die helle Variante -be - Gläslibe $\boldsymbol{e}^{16}$ (= ins Gläslein) - möglich wäre, ähnlich wie bei den Optionen Häfiliba oder Häfilibe (= ins Häfilein [Häferlein, Tässchen]). Bei Stämmen mit dunklen Vokalen - wie es der aus nur ausschließlich dunklen Vokalen bestehende Diphthong [ua] im folgenden Beispielwort belegt - ist hingegen nur die Suffixvariante -ba zulässig; vgl. z.B. Kruagba (= in den Krug). Als aktuellen Trend kann man jedenfalls erkennen, dass die dunkle Variante - $\boldsymbol{b} \boldsymbol{a}$ an Terrain gewinnt.

Die Auseinandersetzung mit diesem Beleg und anderen ähnlichen Redebeispielen provoziert die Frage, in welchen Fällen doppelte Markierungen grammatischer Beziehungen bevorzugt werden. Eine nahe liegende Hypothese wäre, dass solche Phänomene besonders dann auftreten, wenn ein entsprechendes Strukturmuster auch in der Basissprache, d.h. dem deutschen Basisdialekt, in irgendeiner Weise vorhanden ist. Das könnte die Transferenz fördern. Belegsatz Nr. (3) hätte ja auch in der Basisvarietät im unilingualen Modus mit hinein enden können, etwa: Schütte [eigentlich: Fülle] es mir ins Gläslein hinein. Das ungarische Illativsuffix steht mithin an der Stelle des (allerdings vom Substantiv getrennt zu schreibenden) Lokaladverbs hinein. Da es sich gewiss nicht um ein unikausales Phänomen handelt, müsste ein wirklich stichhaltiges heuristisches Erklärungsparadigma wohl zugleich mehrere Argumente von verschiedenen Ebenen heranziehen. Möglicherweise kann man neben Erklärungen der Sprachökonomie - auch argumentieren, dass derartige grammatische Transferenzen eher an strukturell komplexen und kognitiv schwierigen Stellen auftreten.

Eine doppelte Kennzeichnung grammatischer Relationen liegt auch in qualitativ andersartigen Fällen vor. Beispielsweise reihen viele Belege zwei Suffixe - ein ungarisches und ein deutsches - aneinander:

(4) Ȧisi Náchpr seand's gse:i, s Leiéks. (SD: Unsere Nachbarn sind es gewesen, des [= die] Leis.)

Der Familienname lautet Lei, er wurde zuerst mit dem ungarischen Kollektivbezeichnungssuffix -é $\boldsymbol{k}^{17}$ versehen, dann zusätzlich auch auf Deutsch mit -s suffigiert. Diese Erscheinung gilt in Hajosch keineswegs als Ad-hoc-Auffälligkeit, sondern gehört im Ortsdialekt zu den üblichen, ganz und gar gängigen sprachlichen Formen. Man könnte sogar sagen, dass sich -éks als neues (hybrides) Suffix etabliert hat; wie die Beispiele belegen, kann es sowohl deutschen als auch ungarischen Namen angefügt werden, vgl. ván $s$ Nagyéks ('von des [= den] Nagys').

\footnotetext{
${ }^{16}$ Dieses Lexem war früher in den Formen klázli, glászli, glazli, kalázli und kelázli als deutsches Lehnwort bairisch-österreichischer Provenienz auch im Ungarischen regional recht verbreitet. Die ungarische Version ist vor allem durch Auflösung der anlautenden Konsonantenhäufung und durch Dissimilation entstanden (vgl. BENKÖ 1993: 760 und KISS 2001: 215).

${ }^{17}$ Im Ungarischen gibt es eine spezielle Art von Kollektiva, bei der es sich um eine Bildung pluraler Substantive handelt, die eine Gruppe bezeichnen, zu der das betreffende Individuum gehört. Basen sind dabei vorrangig Eigennamen und Personenbezeichnungen. Folglich hat man es hier mit einem ungarischen QuasiWortbildungssuffix zur Markierung von Kollektiva zu tun, das analog zu possessiven Personalsuffixen des Ungarischen an Stämme tritt (vgl. KERESZTES 1999: 74). TOMPA (1972: 109) stuft es klar als „Ableitungssuffix“ ein, nach KENESEI/VAGO/FENYVESI (1998: 353 f.) ist indessen sein Status als Derivationsaffix nicht eindeutig, da es u.U. auch als Flexionssuffix qualifiziert werden kann. Weitere Beispiele: Takácsék (= die Familie Takács), ähnlich wie im Deutschen das Suffix $-s$ in Formen wie Müllers (= die Familie Müller); igazgatóék (= der Direktor und die Seinen).
} 
Für die doppelte (d.h. bilinguale) Kennzeichnung ein und derselben grammatischen Funktion - sie ließen sich auch als eine duale Kodierung auffassen - konnte also eine Vielfalt von Belegen unterschiedlicher Art eruiert werden, z.B.

(5) S Gealt ischt odiván. (SD: Das Geld ist hin [ist]; 'ist' wird also zweimal ausgedrückt.)

Das zusammengesetzte Verb odiván - standardungarisch: odavan - ('ist hin/verloren') wurde von der Sprecherin nicht analysiert, sondern wie ein Simplex gehandhabt und syntaktisch (noch einmal) mit dem deutschen ist verknüpft.

Hybride Präpositionalgefüge sind in diesem Zusammenhang gleichfalls interessant:

(6) Ma seand mit am Autóval uf Mischka gfahra. (SD: Wir sind mit dem Auto auf [nach] Miske gefahren.)

Ähnlich wie bei Beleg Nr. (3) gehen hier diesmal eine dativische Präpositionskonstruktion des Deutschen (mit dem Auto) und ein Instrumentalsuffix des Ungarischen (-val) eine Symbiose ein. Viele Belege unterscheiden sich von dem obigen strukturell dadurch, dass das Substantiv an der Gelenkstelle der hybriden Fügung eindeutig ungarischer Provenienz ist:

(7) Sie hàt de:s älts uf d Mérlegre tau. (SD: Sie hat das alles auf die Waage getan [= gelegt].)

In diesem Satz wird der Ansatz ${ }^{18}$ eines deutschen akkusativischen Präpositionalgefüges mit einem ungarischen Sublativsuffix (-re) verknüpft.

(8) E:scht gega Mà argis seam:a vàn dr Lagziból hu:eikàm:a. (SD: Erst gegen Morgen sind wir von der Hochzeit heimgekommen.)

Hier tritt zum Ansatz eines dativischen Präpositionalgefüges ein Elativsuffix ${ }^{19}$ (-ból $)$ des Ungarischen hinzu.

(9) Uhna Ändrlais: ha:ud sie bis Reggelig tanzed. (SD: Ohne Unterlass haben sie bis zum Morgen getanzt.)

Im obigen Satz wird der Ansatz eines deutschen Präpositionalgefüges mit einem ungarischen Terminativsuffix ${ }^{20}$ (-ig) verbunden.

Auch bei Personennamen sind solche dualen Kodierungen möglich, wie beim Item Nr. (10), wo der Ansatz eines deutschen dativischen Präpositionalgefüges und ein ungarisches Allativsuffix (-hoz) aufeinander treffen.

(10) I: han 's die Kendr äl:awà il mes:a saga, sie sol:id it: na: zum Bandihoz. (SD: Ich habe den Kindern allweil müssen sagen, sie sollen nicht hin zum Bandi [ungarischer männlicher Vorname in Koseform].)

In den vorstehenden hybriden Belegen gelten die thematisierten deutschen und ungarischen Formen als reguläre Entsprechungen voneinander, z.B. die deutsche Präposition $z u$ und das ungarische Allativsuffix -hoz. Indes gibt es sogar Fälle dieser Art von Hybridität, in denen die jeweiligen Sprachmittel keine regulären zwischensprachlichen Äquivalente sind, z.B. ván $d r$

\footnotetext{
18 „Ansatz“, weil ja das Nomen ungarischer Provenienz ist, sodass man m.E. kaum von einem herkömmlichen deutschen Präpositionalgefüge sprechen kann.

${ }^{19} \mathrm{Im}$ Ungarischen stellt eine Elativ-Relation eine Ortsbestimmung des inneren Raumes dar.

${ }^{20}$ Der Terminativ bezeichnet eine Orts- und Zeitgrenze.
} 
TSZ által (d.h. „,von der TSZ által“ - TSZ bedeutet $L P G$ und die ungarische Postposition által entspricht der deutschen Präposition durch).

Mitunter entstehen hochkomplexe hybride Suffixkonglomerate, vgl.:

(11) Äl:igi hock:id den:a en iahri Stubanáikban änd teand Tévé guck:a. (SD: Alle hocken drin in ihren Stuben und tun TV gucken.)

Im obigen Beleg wird nicht nur die Ortsbestimmung (die Präposition en 'in' + das Inessivsuffix -ban 'in'), sondern auch das Possessivverhältnis (Possessivpronomen iahri 'ihre' + Pluralzeichen des Besitzes - $i$ ) und der Plural (Possessivpronomen iahri 'ihre' + Pluralzeichen $\boldsymbol{- k}$ ) doppelt markiert.

Manche der erörterten bilingualen Eigentümlichkeiten (z.B. die zusätzliche Suffigierung beim Substantiv in einem Präpositionalgefüge, wie im Beleg Nr. 3) sind ungemein spannend und verdienen eine besondere Hervorhebung. Wären sie doch beim Kontakt von zwei flektierenden indogermanischen Sprachen nicht denkbar, denn bekanntlich beruhen die verschiedenartigen Kontakt-, Interaktions- bzw. Konvergenzphänomene - außer auf vielfältigen psycho-, neuro-, sozio- und pragmalinguistischen Faktoren - im Wesentlichen auf den strukturellen (typologischen) Eigenheiten und Möglichkeiten der miteinander in Berührung stehenden Sprachen. Über diese Zusammenhänge fehlen noch eingehendere empirische Untersuchungen hinsichtlich des arealen Kontaktes des Deutschen mit nichtflektierenden Sprachen. Zu erwähnen ist aber eine kleinere Arbeit von TEKINAY (1987) über eine zwar soziolinguistisch ganz anders gelagerte und geartete Sprachenkontaktkonstellation, die aber sprachtypologisch meinen Untersuchungen nahe steht. TEKINAY wertete einige leider nicht sehr materialreiche - Sprechproben türkischer Arbeitsmigranten in Deutschland aus und hat in deren von deutschen Transferenzen geprägtem Türkisch eigentlich wenig sprachtypologisch Spektakuläres gefunden. Die Autorin brachte die Möglichkeit und Problematik der Sprachtypologie nicht einmal zur Sprache. Lediglich eine einzige Beobachtung könnte eventuell von Relevanz sein: das Vorkommen doppelter Pluralendung (deutsch-türkisch) in kinder/ler (1987: 100). ${ }^{21}$ Weitere nennenswerte Befunde, etwa Beispiele für eine zweifache Kennzeichnung ein und derselben grammatischen Beziehung mit kategorial unterschiedlichen Mitteln (wie etwa in meinem Beleg Nr. 3 o.ä.), kommen in dieser Publikation nicht vor. Folglich stellt die Aufdeckung von Kontakten des grundsätzlich flektierenden Deutschen mit dem im Wesentlichen agglutinierenden Ungarischen ${ }^{22}$ unter sprachtypologischen Gesichtspunkten m.E. Neuland dar und ist von herausragender wissenschaftlicher Attraktivität.

Anhand der durchgeführten Analyse wurde offenbar, dass lexikalisch-semantische Transferate aus dem Ungarischen deutsche Suffixe erhalten können. Diesbezüglich liefert, vom

\footnotetext{
${ }^{21}$ Einige strukturell ähnliche deutsch-türkische Belege findet man ferner bei KALLMEYER/KEIM/ASLAN/CINDARK 2002: 7). Für doppelte Pluralbildung kann man allerdings auch in typologisch einander weitgehend ähnlichen Sprachen Belege finden; vgl. z.B. Seitens < pages in (kanadisch-) englisch-deutscher Konstellation (HUFEISEN 1995: 247) oder die Ohrens < die Ohren + ears (HOFFMANN 1997: 106). Im Gegensatz zu meiner Transferenz-Auffassung sieht NAVRACSICS (1999: 140) in diesem Phänomen eine Kode-Umschaltung auf der Ebene der Morphologie.

${ }^{22}$ KIEFER (1999) weist plausibel nach, dass das Ungarische keine rein agglutinierende Sprache ist, die Agglutinierung aber eines seiner wesentlichen Charakteristika darstellt. SCHMIDT (1998: 998) charakterisiert das Gegenwartsdeutsch durch ererbte synthetisch-flektierende und neu entwickelte analytisch-isolierende Merkmale; zu weiteren sprachtypologischen Tendenzen vgl. ROELCKE (2002: 259 ff.)
} 
morphologischem Standpunkt aus betrachtet, in erster Linie die Graduierung von Adjektiven aufschlussreiche Fälle zwischensprachlicher Kontaktphänomene.

Manchmal erfolgt eine Transferenz bereits graduierter ungarischer Adjektive im Komparativ, z.B.:

(12) En okosabb gà it's en Ha:josch: it: wia ear. (SD: Einen Klügeren gibt's in Hajosch nicht wie er [eigentlich: als ihn].)

Aus dem Ungarischen transferierte Lexeme können aber im Komparativ auch nach dem deutschen Muster und mit deutschen Steigerungssuffixen versehen werden, z.B.:

(13) He:it ischi é:brar:. (SD: Heute ist er munterer < aus ungar. éber 'munter'.)

Beim Superlativ wird in deutschen Sätzen hin und wieder auf Adjektive ungarischer Provenienz in der im Ungarischen üblichen komparierten Form zurückgegriffen. Belege gibt es sowohl für den (a) attributiven als auch für den (b) prädikativen Gebrauch:

$\mathrm{zu}(\mathrm{a})$ :

(14) Hàscht scha wiedr dr legolcsóbb Pul:ofr ka:uft? (SD: Hast [du] schon wieder den billigsten Pullover gekauft?)

$\mathrm{zu}(\mathrm{b})$ :

(15) D Elefanta mes:id doch am legerösebb se:i. (SD: Die Elefanten müssen doch am stärksten sein.)

In ihrer Mehrzahl bekommen die Adjektive oft das übliche ungarische Pluralzeichen $-\boldsymbol{k}$, z.B.:

(16) Sie weand jä die legelökelöbbek se:i. (SD: Sie wollen ja die Vornehmsten sein.)

Hier nehmen die ungarischen Lexeme legerösebb ('stärkst-'), legelökelöbbek ('vornehmst-'), legutolsók ('letzt-') usw. jeweils eine adjektivische Gestalt an. Im Rahmen von hybriden Superlativformen können aber auch Adverbien ungarischer Herkunft vorkommen:

(17) And wen: s Fuir em legjobban bren:t, nách tuat ma d Krämpi:ara ne:i. (SD: Und wenn das Feuer am besten brennt, dann tut man die Kartoffeln hinein.)

Merkwürdigerweise werden mitunter deutsche Adjektive im Superlativ doppelt, d.h. sowohl ungarisch (mit dem Präfix leg-) als auch deutsch (mit dem Flexionssuffix -st) graduiert:

(18) Sie seand stark arm, abr die legiarmischti seand doch s Wendmach:rs gse:i. (SD: Sie sind stark [= sehr] arm, aber die ärmsten sind doch die Wendmachers gewesen.)

Solche Belege hybrider Flexionsparadigmen demonstrieren, dass im Sprachenkontakt auch stark gebundene Morpheme transferiert werden können. Im Elativ ist die Verbreitung der hybriden Formen bzw. Bildungsarten ebenfalls sehr groß.

Für die Transferenz bereits auf Ungarisch graduierter Formen lässt sich Beleg Nr. (19) anführen:

(19) S legislegfontosabb ischt jetz:, das: da zum Doktr gàhscht. (SD: Das Allerwichtigste ist jetzt, dass du zum Doktor gehst.) 
Deutsche graduierte Adjektive können mit ungarischen Präfixen verknüpft werden, was zu einer dualen Komparation führt:

(20) S legislegarmseligscht Häusli ischt en dr Alta Gas: gstanda. (SD: Das allerärmste Häuschen ist [= hat] in der Alten Gasse gestanden.)

Andererseits gibt es auch das Gegenteil, wenn sich also die doppelte Markierung darin zeigt, dass bei Elativkonstruktionen graduierte Adjektive ungarischer Provenienz mit der deutschen Vorsilbe aller- verbunden werden:

(21) S äl:rfontosabb ischt jetz:, das: da.... (SD: Das Allerwichtigste ist jetzt, dass du...).

3.3. Aus meinen Belegtypen ist zweifellos eine sprachlich-strukturelle Vielfalt von Erscheinungen zu ersehen. So kann man etwa erkennen, dass manchmal deutsche Verben nach ungarischem Muster konjugiert werden (vgl. Nr. 2). Aber noch öfter werden aus dem Ungarischen transferierte Verben nach der Bildungsart des Deutschen suffigiert:

(22) Ear hàt karambolozned ànd sie ischt béna wà:ara. (SD: Er hat karamboliert [= hatte einen Unfall] und sie ist [deshalb] gelähmt.)

Oft verwandeln sich auf diese Weise besonders ungarische onomatopoetische Wörter und Vokabeln mit spezifischer Bedeutung, Konnotation und/der Lautung sowie Realienbezeichnungen bzw. Bezeichnungsexotismen (oder „kulturspezifische Wörter“23 im Sinne von GODDARD/WIERZBICKA 2003: 148 ff.) zu deutschen flektierten Verben oder Partizipien, vgl.:

(23) S Was:r tuat lotsch:ala. (SD: Das Wasser [tut] plätschern < aus ungar. locsol/locsog.)

Im Hintergrund des Syntagmas ischt vacseszned verbirgt sich wohl das - transferierte und auf Deutsch flektierte (ver- $+-t$ ) - saloppe ungarische Verb cseszik (eigentlich 'bumsen') bzw. präfigiert elcseszik ('verpfuschen'), genauer die Phrase el van cseszve ('ist verpfuscht'), vgl.

(24) Me:i Gwand, was d Nähdri gmacht hàt, ischt guat vacseszned ànd $i$ : han's ihnr gse:it, wia sie's mach:a sol: (SD: Mein Gewand, was [das] die Näherin gemacht hat, ist gut [= richtig] verpfuscht und ich habe es ihr gesagt, wie sie es machen soll.)

Aus der engen Symbiose der beiden kontaktierenden Sprachvarietäten resultiert oftmals eine enge Verzahnung der deutschen und der ungarischen Grammatik, indem die beiden Sprachen stark miteinander „kooperieren“. So kann bei - echten wie unechten - reflexiven Konstruktionen das (deklinierte) Reflexivpronomen aus der einen und das (ebenfalls flektierte) Hauptverb aus der anderen Sprache stammen:

(25) I: ha:n mi: so szégyellnid. (SD: Ich habe mich so geschämt < ungar. szégyell), bzw.:

(26) Sie hat si so összegömbölyödned. (SD: Sie hat sich so zusammengeknäuelt < ungar. összegömölÿ̈dik.)

Hybriditäts-Prozeduren sind mitunter derart komplex, dass bei manchen Mischprodukten bereits die Sprachenzuordnung problematisch ist. Es wird deutlich, dass die Sprachenkontakte nicht simple mechanische Mischungen herbeiführen, bei denen alle Komponenten stets

\footnotetext{
${ }^{23}$ Oder m.E. zumindest ,kulturtypische Wörter“.
} 
eindeutig den beteiligten Sprach(varietät)en zugeordnet werden können. So fordern die Belege Nr. (27) und (28) diesbezüglich zum Nachdenken auf:

(27) D Luckri szétschäar:ázta a Neaschtot. (SD: Die Gluckhenne [= Glucke] zerscharrte das Nest.)

Dieser Satz beginnt noch eindeutig im Dialekt (mit einem deutschen Artikel), die anderen Elemente sind hingegen nur in lexikalischer Hinsicht deutsch. Das Korpus enthält eine Vielzahl solcher hybriden Belege, in denen innerhalb einer Wortform eine mehrfach gemischte Morphemstruktur vorliegt, z.B. ungarisches Präfix + deutscher Stamm + ungarisches Suffix bzw. deutsches Präfix + ungarischer Stamm + deutsches Suffix.

Der folgende Satz (28) kann bereits sowohl aus morphologischer als auch aus syntaktischer Sicht als ungarisch betrachtet werden.

(28) Schälázd meg az Äpf:lt! (SD: Schäle den Apfel!)

Im Gegensatz zum Beispiel Nr. (27) ist hier auch schon der bestimmte Artikel ungarisch. Diese und ähnliche sprachliche Produkte demonstrieren plastisch die Dynamik und die Prozessualität der erörterten Sprachenkontaktwirkungen.

3.4. Es ist wichtig zu konstatieren, dass sich die lexikalischen Transferenzen in morphologischer Hinsicht in vielen Fällen nicht vollständig in den deutschen Kotext integrieren und dadurch mitunter auffällige morphologische Diskrepanzen erzeugen. Global betrachtet, kann man für solche Erscheinungen mehrere Gründe anführen: Beispielsweise kann ein Lapsus beim Sprechen vorliegen oder es handelt sich möglicherweise darum, dass die morphologische Struktur des anderssprachigen Sprachzeichens für den deutschsprachigen Sprecher nicht erkennbar ist.

Bei meinem Material kommen die substantivischen Transferenzen aus dem Ungarischen grundsätzlich als Grundform im Nominativ Singular in den deutschen Dialekt. Dort nehmen sie oft weder die ungarischen noch die deutschen (a) Plural- und/oder (b) Kasusendungen (besonders im Akkusativ) an.

Vgl. zu (a):

(29) Dot: ha:ud a:u Telepes gwuhnt. (SD: Dort haben auch Ansiedler ${ }^{24}$ gewohnt.)

$\mathrm{zu}(\mathrm{b})$ :

(30) Die Kendr ka:ufa ma großi Fürdölepedö. (SD: Den Kindern kaufen wir große Badetücher.)

Der Artikelgebrauch zeigt ebenfalls kontaktbedingte Besonderheiten. In vielen Fällen bleibt vor allem der unbestimmte - Artikel weg:

(31) En aisr Gas: mach:id sie Alap. (SD: In unserer Gasse machen sie [= macht man] ein Fundament.)

Besonders oft kommt dies bei den ungarischen Varianten von im Standarddeutschen existierenden „Fremdwörtern“" vor, z.B.

\footnotetext{
${ }^{24}$ Unter Telepes ('Ansiedler') verstehen die Hajoscher Ungarndeutschen die aus der Slowakei, dem vormaligen Oberungarn, zwangsweise hierher umgesiedelten Ungarn. Das Wort ist auch z.B. bei SCHWEDT (1995: 36) belegt.
} 
(32) Me:in Vat:r hät Infarktus ghät: (SD: Mein Vater hat [einen] Infarkt gehabt.)

Die Häufigkeit und Geläufigkeit der Transferenz mehr oder weniger homophoner bzw. homomorpher Lexeme mit identischer Bedeutung ist im Hinblick auf andere Sprachenpaare in der Forschungsliteratur bereits dokumentiert worden. CLYNE (1992) erklärt das z.B. mit einem genuin psycholinguistischen Ansatz, nämlich mit einer „,innersprachlichen Auslösung“ - gerade infolge einer morphologischen und semantischen Äquivalenz der beiden Versionen in den kontaktierenden Sprachen.

In anderen Fällen findet sich bei den ungarischen Worttransfers ein unbestimmter Artikel auch dann, wenn sowohl im Standarddeutschen als auch im Ungarischen Nullartikel steht:

(33) Die Hasa mus: ma an Táp ka:ufa. ${ }^{25}$ (SD: Den Hasen [= Kaninchen] muss man ein [sic!] Futter kaufen.)

3.5. Wohl unter dem Einfluss des Ungarischen finden sich manche Belege für das gemeinsame Auftreten eines bestimmten Artikels und eines Possessivpronomens in der Funktion als Determinanten des Substantivs (vgl. die meine Uhr im folgenden Beleg):

(34) Di me: i Uhr set: ma a:u ne:ineam:a ànd an Elem ne:imach:a la:u. (SD: [Die] meine Uhr sollte man auch hineinnehmen [zum Uhrmacher] und eine Batterie hineinmachen $\operatorname{lassen}^{26}\left[=\right.$ einsetzen].) $^{2}$

3.6. Die morphosyntaktisch relevanten Kontakteinflüsse des Ungarischen können mitunter von ungemeiner Subtilität sein. Aus meinem Datenkorpus geht z.B. eine vielschichtige Problematik des Pluralgebrauchs hervor. Aus dem Ungarischen transferierte Lexeme können in einem deutschsprachigen Kontext ohne Weiteres im Plural stehen, vgl.:

(35) D Csibék seand äl: em Gata danda. (SD: Die Küken sind alle im Garten drunten.)

Dessenungeachtet stehen Substantive ungarischen Ursprungs sowohl nach bestimmten als auch nach unbestimmten Zahlwörtern nicht im Plural, vermutlich, weil im Ungarischen das Bezugswort nach seinem numeralischen Attribut stets im Singular verwendet wird, vgl.:

(36) Zwua Pénztárca seand uf am Tisch doba gleaga. (SD: Zwei Geldbörse [sic!] sind auf dem Tisch droben gelegen.)

(37) A paar Palacsinta han i gmacht. (SD: Ein paar Palatschinke [sic!] habe ich gemacht.)

3.7. Weitere recht bemerkenswerte - um nicht zu sagen: faszinierende - interlinguale Erscheinungen kann man bei einigen Formen der Hybridisierung in der Wortbildung entdecken. Diese Eigenheiten besitzen auch deswegen eine komplexe und weit reichende Bedeutung, weil sich die kommunikativen Bedürfnisse sowohl des Individuums als auch des Soziums wohl am prägnantesten in der Wortbildung artikulieren. Die im Blickpunkt stehenden Hybridisierungen führen eine zwischensprachliche Grammatikalisierung herbei.

Extrem produktiv, hochvital und kreativ sind also die mit der ungarischen Vorsilbe akárentstandenen kompakten und komprimierten „ungarndeutschen“ Pronominaladverbien. Das

\footnotetext{
${ }^{25}$ Es ist nicht ausgeschlossen, dass der Sprecher mit dem Ausdruck an Táp ('ein Futter') im gleichen Sinne eine Maß- bzw. Mengenbezeichnung gemeint hat, wie etwa in egy zsák táp ('ein Sack Futter'); deshalb verwendet er den unbestimmten Artikel.

${ }^{26}$ Schon die Formulierung deutet auf ein ungarisches Modell hin, denn im Ungarischen wäre in einem solchen Satz beletetet (= hineintun/-machen lassen) üblich.
} 
entsprechende Muster des Ungarischen hat im deutschen Dialekt regelrecht einen Wortbildungsimpetus in Gang gesetzt, d.h. es wirkt paradigmenbildend und führt zu ganzen Wortbildungsreihen (zu dieser Terminologie siehe FLEISCHER/BARZ 1995: 69); vgl.:

(38) Ak:a:rwian i: 's wil:, 's weat: doch it: guat. (SD: Wie ich es auch immer will, es wird doch nicht gut.)

Die zu Grunde liegenden durch Zusammensetzung konstruierten ungarischen konzessiven Komposita werden vom deutsch-ungarischen bilingualen Sprachträger reetymologisiert, wodurch ihre Motivation klar in Erscheinung tritt: Die Vorsilbe akár-, die etymologisch mit der Konjunktion akár verwandt ist (< akar,wollen'), wird aus der Zusammensetzung isoliert und mit den deutschen Fragepronomina wohin und wie verknüpft. Es gibt auch weitere Formen wie ak:a:rwas, ak:a:rmo (<wo) etc. Die mit akár- gebildeten Zusammensetzungen verankerten sich übrigens schon sehr früh im sprachkommunikativen Repertoire der Ungarndeutschen: Selbst einsprachige Personen, die des Ungarischen in keiner Weise kundig waren, haben sich regelmäßig im unilingualen Gesprächsmodus dieser hybriden Konstruktionen bedient. Diese Pronominaladverbien scheinen übrigens in diversen wahrscheinlich in allen - ungarndeutschen (bzw. z.T. sogar in sämtlichen „donauschwäbischen“) Gegenden im Karpatenbecken gängig zu sein. So findet man Belege in unterschiedlichen Quellen, z.B. im Beitrag von KNAB (1997) über die Mundart des Nachbardorfes Nadwar/Nemesnádudvar. ${ }^{27}$ Ebenso in KNECHTs Publikationen (1999: 354, 2001: 74 und 96 f.) über sathmarschwäbische Dialekte im heutigen Rumänien. HUTTERER (1993: 160) weist nach, dass diese akár-Ausdrücke mitunter ,auch in mehrheitlich nicht ungarischer Umgebung“" gängig sind. Selbst in Dialektwörterbüchern findet man sie lemmatisiert. ${ }^{28}$

Neben den genannten Formen fanden sich in meiner Hajoscher Datenbasis Konstruktionen wie: ak:a:rwa:s (akár + was), ak:a:rwear (akár + wer), ak:a:rmo (akár + wo), ak:a:rwen: (akár + wann), ak:a:rwiavl (akár + wie viel), ak:a:rwa:s fa:r a (akár + was für ein), ak:a:r ván mo/ak:a:r ván mohear/ván ak:a:rmo (akár + von wo/akár + von woher/von + akárwo). Einige Beispiele:

(39) Ak:a:rwear das: kàm :t, las: 'a re:i! (SD: „Akárwer“ dass kommt, lass [ihn] rein!, d.h. Wer auch immer kommt, ...)

(40) Ak:a:rwiavl Leut das: kàm:ed, so ha:ud sie Platz: (SD: „Akár wie viel“ Leute dass kommen, so haben sie Platz, d.h. Wie viele Leute auch immer kommen, sie werden einen Platz finden.)

(41) Ak:a:rwas fä: ar a Weat:r das: kàm:t, abr hack:a mes:a ma ga:u. (SD: „Akárwas für ein“ Wetter kommt, aber hacken müssen wir gehen, d.h. Was für ein Wetter auch immer kommt, ...)

Die Flexionsmöglichkeiten dieser hybriden Pronominaladverbien verdienen ebenfalls eine gesonderte Erwähnung: die traditionellen Formen lauten z.B. mit ak:a:rwe:la Lef:l ('mit „akárwelchem“"Löffel'), ván ak:a:rwe:lr Gabl ('von ,akárwelcher" Gabel') oder ván ak:a:rwe:li Mandsna:ma ('von ,akárwelchen“" Männern').

\footnotetext{
${ }^{27} \mathrm{KNAB}$ führt folgende Sätze an: Ich heb net von de' beschte g'lennt, ich war nar so a Mitt'lschilerin, aw'r die, „,akar “ (= gleich) weli Klass' daß ich war, hén die Lehrene mich imm'r allz'fahrt gen g'hatte (1997: 189). Oder: „Akar" (= Gleich) was des war far a' Nazio (1997: 193).

${ }^{28}$ SCHWALM (1979: 30) bringt beispielsweise für Waschkut/Vaskút die Form ,akarwas“ in den Bedeutungen 1. ,was auch immer', ,was ... auch', 2. ,irgendwas'.
} 
Indes gibt es heute schon Doppelformen: Im Maskulinum: ak:a:rwe:la Lef:l/ak:a:r $d r$ we:l Lef:l ('akárwelcher Löffel') bzw. in einem obliquen Kasus mit der Präposition mit zunehmend: ak:a:r mit we:lam Lef:l. Im Femininum: ak:a:r die we:l Gabl ('akárwelche Gabel') bzw. als Präpositionalgefüge mit vàn ('von') zunehmend: ak:a:r ván we:lr Gabl. Im Neutrum: ak:a:rwe:las Mea s:r /ak:a:r s we:l Mea s:r ('akárwelches Messer') bzw. in einem obliquen Kasus mit der Präposition mit zunehmend: ak:a:r mit we:lam Meas:r. Im Plural: ak:a:rwe:li Mandsna:ma ('akárwelche Männer') bzw. als Präpositionalgefüge mit ván ('von') zunehmend: ak:a:r vän we:li Mandsna:ma. Meine Untersuchungsergebnisse legen die Vermutung nahe, dass gegenwärtig Formen wie im Beleg Nr. (42), die der sprachlichen Konstruktionsweise des Ungarischen strukturell näher stehen, häufiger auftreten als die „traditionellen“:

(42) Ak:a:r en we:las Haus nei, das:'r kam:a ischt, d Stuba ischt voll gleaga mit Stroh. (SD: [Am Heiligabend] „Akár“" in welches Haus hinein, dass er gekommen ist, die Stube ist voll gelegen mit Stroh).

Auch diese Fälle zeugen von einem hohen Maß an Flexibilität, Dynamik und Prozessualität von sprachlichen Kontaktmechanismen.

Der im binnendeutschen Standard dieser Konstruktion entsprechende Adverbialsatztyp ist in der Grammatik auch hinsichtlich seines Status problematisch. Er wird z.B. von KÖNIG/EISENBERG (1984: 314 f.), von den Konzessivsätzen getrennt, als „Irrelevanzkonditionale“ behandelt. Meiner Ansicht nach kommt es deswegen zur Übernahme der Vorsilbe akár- und zu verschiedenen damit gebildeten Komposita, weil die als Muster dienende ungarische Konstruktion sprachlich viel einfacher, ökonomischer und transparenter ist als die analytischen - und recht komplizierten ${ }^{29}$ - Ausdrucksweisen des Deutschen $(w$ Fragewort + auch immer + Nebensatz), sodass durch die Hybridisierung die kognitive Verarbeitung vereinfacht wird. Der Transparenz fällt eine besondere Bedeutung zu, weil sie dem kognitiven Prozess entgegenkommt, was wiederum den zwischensprachlichen Transfer begünstigt. Andererseits gilt auch, dass - wie in der Systemtheorie LUHMANNs (2000: $48 \mathrm{ff}$. und 236) - jede Komplexitätsreduktion immer eine Komplexitätssteigerung nach sicht zieht.

Ähnlich gelagerte Beispiele kann man im Hajoscher Ortsdialekt mehrfach finden. Der etymologische Hintergrund der hybriden Demonstrativpronomina

ugyande:a/ugyandi:a/ugyande:s (SD: derselbe/dieselbe/dasselbe) und ugyanseal: (SD etwa: dasselbe dort), mit denen die Identität einer Person oder eines Gegenstandes mit einer/einem zuvor genannten ausgedrückt werden kann, ist besonders aufschlussreich. Nach ihrer Bildungsart können sie als Lehnverbindungen charakterisiert werden und gehen auf ungar. ugyanez und ugyanaz zurück. Diese ungarischen Prototypen sind sprachhistorisch aus dem verstärkenden und intensivierenden Adverb ugyan und dem Demonstrativpronomen $\boldsymbol{e z}$ bzw. $\boldsymbol{a z}$, durch Zusammenrückung entstanden. Das erste Glied ugyan hatte eine verstärkende Funktion und ging aufgrund des häufigen gemeinsamen Auftretens mit manchen Pronomina (z.B. $\boldsymbol{e} z-$ dt. 'dies') oder Adverbien (z.B. ott - dt. 'dort') im Sprachusus eine Einheit ein. So entwickelte sich im Laufe der Zeit aus einer ursprünglichen Wortverbindung ein Kompositum. Die in den Hajoscher Belegen auftretenden Amalgamierungen von ungar. ugyan $+\mathrm{dt}$. der/die/das und ungar. ugyan $+\mathrm{dt}$. seal: (ein Demonstrativum aus selb, das einen etwas weiter entfernten Bezugspunkt bezeichnet, wie 'jener') entsprechen semantisch etwa

\footnotetext{
${ }^{29}$ Unlängst hat sich LEUSCHNER (2000) mit diesen „Irrelevanzpartikeln in Nebensätzen mit w-auch/immer ausführlich auseinander gesetzt und diesen Konstruktionen hohe Komplexität wie auch eine ,(scheinbar) unübersichtliche Formenvielfalt“" (2000: 342$)$ attestiert.
} 
dem standarddeutschen Demonstrativum derselbe/dieselbe/dasselbe (das seinerseits aus dem bestimmten Artikel und dem alten Pronomen selb- zusammengesetzt ist). Vgl.

(43) Ugyande:a Zwet:r hàt sie ka:uft. (SD: Ugyanden [= denselben] Pullover hat sie gekauft.)

(44) Ugyandi:a Blus ha:n i: miar rausgsu:acht. (SD: Ugyandie [= dieselbe] Bluse habe ich mir ausgesucht.)

(45) Dot: ha:n i: ugyande:s gsi:ah. (SD: Dort habe ich ugyandas [= ebendas] gesehen.)

Die Analyse zeigt, dass sich in den obigen Belegen die „ungarndeutsche“ Wortbildung - unter Einbeziehung deutscher und ungarischer Elemente - auf der Basis von Wortbildungsstrukturen des Ungarischen (und nicht des Deutschen!) vollzieht. Trotzdem finden aber dabei - auf eine spezifische Weise - die Eigenheiten beider Sprachvarietäten größtmögliche Berücksichtigung: Im Ungarischen steht normalerweise ugyanaz a (wörtlich: 'derselbe der') etc., d.h. dem substantivischen Demonstrativpronomen folgt der bestimmte Artikel. (Hier wird auch ein sprachtypologischer Unterschied deutlich: Im Ungarischen drückt der Artikel nur Bestimmtheit aus, im Deutschen auch das Genus.) In den ungarndeutschen Verwendungsbelegen steht dagegen das hybride Kompositum als Substitut für das Demonstrativpronomen plus den Artikel des Ungarischen gemeinsam, d.h. die ungarndeutsche Version spart sich den Artikel; der Artikel wird nur einmal (im Kompositum) gesetzt. Damit wird - trotz des ungarischen Wortbildungsmodells - den sprachlichen Gesetzmäßigkeiten des Deutschen Genüge getan. Beachtenswert ist auch die Parallelität zwischen den binnendeutschen standardsprachlichen und den kontakt-induzierten ungarndeutschen Formen in einem weiteren Punkt: Bei bestimmten Präpositionen kommt es im Binnendeutschen zur Trennung von Artikel und selb (vgl. derselbe Augenblick, aber: im selben Augenblick) und in den ungarndeutschen Belegen wird ugyan von der/die/das bzw. seal: (= jener) gleichfalls getrennt, wie von $\mathrm{Nr}$. (46) klar belegt, wobei das zugrunde liegende Modell allerdings eher dem Ungarischen entstammt. Man vgl. ungar. ugyanabban > ugyan in jenem/jeder (von den sprachtypologischen Differenzen einmal abgesehen, denn die ungarische Version ist eine agglutinative Bildung, während die ungarndeutsche mit einem Präpositionalkasus operiert):

(46) Ugyan en sea l:r Salfe:t den:a. (SD: „Ugyan“ in jener [= in eben jener] Serviette drinnen.)

Ungeachtet der ungarischen Vorlage werden aber wieder einmal auch die Gesetzmäßigkeiten und Gepflogenheiten des Deutschen berücksichtigt. Im Ungarischen wird nämlich das Kasussuffix zweimal verwendet (vgl. ugyanab ba $\boldsymbol{n}$ a szalvétá $\boldsymbol{b}$ a $\boldsymbol{n}$ ), während im Deutschen die Kasuskennzeichnung nur im Zusammenhang mit dem Artikel erscheint (vgl. SD: in de r s elben Serviette). Man sieht also: dass die hybride Bildung ugyan en seal:r Salfe:t den:a bis zur Komponente ugyan ungarisch geprägt ist, wobei ugyan wohl lediglich zum Zwecke des Nachdrucks in den ungarndeutschen Ausdruck transferiert wurde. Ansonsten verläuft alles nach den Gesetzmäßigkeiten des Deutschen, so wird die Kasusrelation im Deutschen nicht zweimal gekennzeichnet. Insgesamt ist also das Bildungsprinzip als logisch und nachvollziehbar anzusehen: Lediglich das Verstärkungselement wird aus dem Ungarischen übernommen; im Anschluss daran gelten klar und ohne ungarische Beeinflussung die im Deutschen üblichen Konstruktionsregulierungen.

Wie bei den Belegen Nr. (38) bis (42) mit akár- können auch hier Faktoren wie Kompaktheit und Sprachökonomie eine maßgebende Rolle gespielt haben. Somit weichen meine Betrachtungen von der globalen Feststellung von KNIPF-KOMLÓSI ab, wonach ein „Streben nach Ökonomie, sowie die Komprimierung mehrerer Informationen in einem Wortkomplex 
[...] für die außerhalb des geschlossenen deutschen Sprachraums existierenden Dialekte nicht charakteristisch" sei (2003: 9). Denn zwischen binnendeutsch derselbe und ungarndeutsch ugyander mag zwar unter dem Blickwinkel der Sprachökonomie und Prägnanz kein essenzieller Unterschied bestehen, im Ungarischen - und unter seinem Einfluss auch in den ungarndeutschen Belegen - vermag aber das verstärkende Adverb ugyan auch in Verbindung mit Adverbien ein Kompositum zu bilden, vgl. beispielsweise das Pronominaladverb ugyanott (ott $=$ 'dort'). Dies kann im binnendeutschen Standard nur mit der doch umfangreicheren Wortgruppe am gleichen/an demselben Ort ausgedrückt werden.

Meine Datenbasis wartet auch mit Beispielen für die Kombination verschiedener solcher Konstruktionen auf. Formen wie ugyande:s Bild und de:s näm:lig Bilt (beide: 'dasselbe Bild') sind im untersuchten Dialekt in gleicher Weise üblich, zudem kommt im Sprechusus auch eine bilinguale Kombination der beiden obigen Ausdrücke vor: ugyande:s näm:lig Bilt ('dasselbe Bild'). Vermutlich wollen die Sprecher mit dieser Dopplung ihrer Aussage ein größeres Gewicht verleihen. Gleichfalls finden sich Belege für maskuline und feminine Formen: ugyande:a näm:laga Ma: ('derselbe Mann') bzw. ugyandi:a näm:lig Farb ('dieselbe Farbe').

Kontaktlinguistisch ebenfalls bemerkenswert sind zusammengesetzte hybride Adverbien und Konjunktionen. Beispielsweise dient das „ungarndeutsche“ Adverb hátwen: zum Ausdruck von Eventualität:

(47) Hátwen: dr Gas ausgàht. (SD: Wenn aber das Gas ausgeht.)

Dabei ist hát eine Konjunktion aus dem Ungarischen. Hátwen: gilt als eine Nachbildung des ungarischen Adverbs hátha ('wenn aber, vielleicht'); die Komponente ha entspricht im Deutschen der Konjunktion wenn. Ähnlich nimmt sich das ,ungarndeutsche“ Negationswort dehogyit aus. ${ }^{30}$ Dehogy ist ein ungarisches Negationselement, it ist die Hajoscher ungarndeutsche Version von deutsch nicht. Mit dehogyitt liegt also ein Reflex von ungarisch dehogynem (< dehogy ['dexodj] = 'ach nein/mitnichten' + nem $=$ 'nicht' $)^{31}$ etwa im Sinne von 'und ob', als positive Antwort auf eine negativ gestellte Frage vor.

Ferner kann die ungarische subordinierende Konjunktion hogy im untersuchten

Dialektdiskurs u.U. mit deutschen Konjunktionen kombiniert bzw. zusammengefügt werden, z.B. hogy wen: ('wenn' < ungar. hogyha - als Konjunktion von Konditionalsätzen - wörtlich 'dass wenn'). Die Sprecher nehmen diese Fälle wohl kaum als eine Art zwischensprachliche Reduplikation oder als Pleonasmus wahr, vielmehr folgen sie automatisch dem Konstruktionsschema des Ungarischen, in dem die Fusion der zwei Konjunktionen - hogy ('dass') bzw. ha ('wenn') - als eine echte subordinierende Konjunktion gilt:

(48) An d u:eini ha:ud näch scha gse:it, hogy wen: ma Bu:einr fendt, mit seal:i sol: ma s Kreuz mach:a. (SD: Und einige haben dann schon gesagt, dass wenn man Knochen findet, mit jenen [= denen] soll man das Kreuz machen.)

Auf den Konvergenzstimulus der Referenzsprache Ungarisch hin können deutsche Konjunktionen ihre ursprüngliche Bedeutungsstruktur bzw. ihre semantisch-pragmatischen Gebrauchsmodalitäten wesentlich verändern. Vgl. etwa das in der binnendeutschen

\footnotetext{
${ }^{30}$ Im Waschkuter Dialektwörterbuch von SchwALM (1979: 331) ist tehodjšnet im Sinne 'warum denn nicht' aus ungarisch dehogyisnem verzeichnet.

${ }^{31}$ Das ungarische $\boldsymbol{h o g} \boldsymbol{y}$ kann mit anderen Satzkomponenten (Negations- bzw. Korrelatwörtern) verschmelzen.
} 
Gegenwartssprache als proportionale Satzteilkonjunktion - als Korrelat zu je - fungierende det:o: (bei GRIMM 1860: II/1034 noch als Adverb gebucht), das die Bedeutung und die Funktion des ungarischen (csak) azért is übernommen hat:

(49) An d det:o: ha:n i's gmacht. (SD: Und zum Trotz habe ich es doch gemacht.)

Man kann also resümieren: Besonders die in den Belegen (38) bis (42) enthaltenen Phänomene sind anschauliche und überzeugende Beispiele für den Transfer stark gebundener morphologischer Elemente. Andererseits ermöglichen sie Einsichten in bestimmte sprachliche und psycholinguale Prozesse bei der Sprachproduktion unter spezifischen

Mehrsprachigkeitsbedingungen, deren Aufdeckung erhebliche theoretische Relevanz besitzt.

Ein besonderer Reiz liegt für interlinguale bzw. interkulturelle Betrachtungen in den Kontaktphänomenen, bei denen der bilinguale Sprecher manche Lexeme zwischensprachlich uminterpretiert und dabei auch einen Wortartwechsel vornimmt, z.B.:

(50) Des Haus ischt ihm it: tetszig gnu:a gse:i. (SD: Dieses Haus ist ihm nicht „tetszik“ genug gewesen, d.h. dieses Haus hat ihm nicht besonders gefallen.)

Hier ist „tetszig“" eigentlich eine ungarische finite Verbform (3. Person Singular) in der Bedeutung '(es) gefällt'. Der zweisprachige Sprecher hat das ungarische Verb tetszik vielleicht wegen seiner Lautgestalt - wie ein deutsches Adjektiv behandelt und das Personensuffix des Verbs des Ungarischen als ein Adjektivsuffix des Deutschen (-ig) empfunden. Somit fand in diesem Beleg eine zwischensprachliche morphologische Transformation von großem Aufschluss statt. In anderen Fällen bewirkte die bilinguale Transformation keinen Wortartwechsel, sondern z.B. eine Deonymisierung von Eigennamen: In Hajosch nennt man in der Niederung den gebundenen, matschigen Boden Scha:rge:s, worin das ungarische Toponym Sárköz (auf Deutsch Scharbruch) steckt, wahrscheinlich, weil in der Sárközer Region, der unmittelbaren Nachbarschaft, dieser Bodentyp vorherrscht, waren doch in der ehemaligen Inselwelt bis zur Regulierung der Donau Mitte des 19. Jahrhunderts Gewässer, Furten und Sümpfe charakteristisch.

3.8. Der durchgreifende Sprachenkontakt macht sich auf rein syntaktischem Gebiet ebenfalls geltend. Die ungarische subordinierende Konjunktion hogy (= dass) ist immer häufiger auch in sonst vollständig deutschsprachigen Sätzen anzutreffen. In solchen Fällen herrscht im Nebensatz allerdings nicht die im binnendeutschen Standard normative Endstellung des Finitums, sondern die Zweitstellung, wie in:

(51) Mit am Apadick: isch a:u so, hogy i:amal isch bes:r, i:amal schle:achtr. (SD: Mit dem Appetit ist es auch so, dass einmal ist [er] besser, einmal schlechter.)

Hätte in diesem Satz die im Ortsdialekt ebenfalls mögliche deutsche Konjunktion das: gestanden, so wäre die Satzgliedstellung wie folgt gewesen: Mit am Apadick: isch a:u so, das: i:amal bes: $r$ ischt, $i$ :amal schle:achtr. (SD: Mit dem Appetit ist es auch so, dass [er] einmal besser ist, einmal schlechter.) Diese Belege zeigen deutlich, dass in der Zweisprachigkeitssituation syntaktische Konflikte zwischen den interagierenden Sprachsystemen in der Regel vermieden werden. Die relativ hohe Frequenz der Transferenz von Konjunktionen u.dgl. lässt sich wohl in kognitiver Hinsicht so erklären, dass viele logische Relationen (z.B. Verknüpfungen) inhaltlich übereinzelsprachlich und daher beim Sprecher weniger an die Realisierung in einer gegebenen Einzelsprache fixiert sind als etwa die autosemantischen Elemente der Sprache. 
Es konnte überdies jedoch beobachtet werden, dass eine für das Ungarische charakteristische Wortstellung häufig in Verbindung mit ungarischen Funktionswörtern auftaucht. Dies ist ein Indikator dafür, dass morphosyntaktische Merkmale meist kontextuell transferiert werden, d.h. dass die ungarischen Lexeme ihre semantischen, morphologischen und syntaktischen Merkmale mitbringen.

Die Konjunktion $s \dot{a} n d r$ (= sondern) existiert in der Hajoscher Mundart zwar noch, ihr Gebrauch nimmt aber zugunsten des ungarischen Pendants rapide ab. So sind alternative Formen möglich: $s \dot{a} n d r$ vs. hanem (= die ungarische Entsprechung für sondern):

(52) Seal: ischt ku:ei Jägrhàus gse:i, was gstanda ischt, hanem a Tschesahäu ${ }^{32}$ gse:i. (SD: Jenes ist kein Jägerhaus gewesen, was [= das] [da] gestanden ist [= hat], sondern ein Feldhüterhaus.)

Der Gebrauch der subordinierenden (alternativen) mehrteiligen Konjunktionen entfaltet zunehmend einen beeindruckenden bilingualen Variationsreichtum. Es konnten z.B. in disjunktiven Satzverbindungen folgende Paare belegt werden: entwedr-odr, vagy - vagy, $\boldsymbol{v a g y}-o d r, o d r-v a g y, o d r-o d r$. Der Kürze halber sollen nur zwei Beispiele angeführt werden:

(53) Vagy ufs Oschtra vagy ufs Fescht hät sie de:s Gwand kriat. (SD: Vagy [= entweder] aufs [zu] Ostern vagy [= oder] aufs [zum] Fest hat sie dieses Gewand gekriegt.)

(54) Vagy vá:ar Mit:rna:cht it: schlafa ken:a odr ná: die zwölfi wach:a. (SD: Vagy [= entweder] vor Mitternacht nicht schlafen können oder nach zwölf wachen.)

Auch die doppelte Negation ${ }^{33}$ breitet sich im Hajoscher Dialekt - wohl in Analogie zum Ungarischen - aus. So kann beispielsweise mit dem Negationspronomen niamr(d) (= niemand) die Negationspartikel it: (= nicht) fakultativ auftreten:

(55) Dáischt niamrd (it:) mitkàm:a mit dr Läicht. (SD: Da ist niemand [+ nicht = fakultatives Element] mitgekommen zum Begräbnis.)

Nach meinen Beobachtungen gibt es zwischen dem Typ der Negation und der Wortstellung eine Korrelation: $\mathrm{Ob}$ beide Negationen möglich sind oder ob lediglich die doppelte Negation zum Einsatz kommen kann, hängt in vielen Kotexten von der Wortstellung ab. Mit der folgenden Wortstellung ist nur die doppelte Negation möglich:

(56) Ni:ana han i: ku:ein gscháida Zwet:r gfända. (SD: Nirgendwo habe ich keinen gescheiten [normalen] Pullover gefunden.)

Bei einer anderen Wortstellung sind beide Negationen denkbar:

(57) En gschäida Zwet:r ha:n i: ni:ana (it:) gfända. (SD: Einen gescheiten [normalen] Pullover habe ich nirgendwo (nicht) gefunden.)

Die verschiedenen Formen mit doppelter Verneinung gewinnen (auf Kosten der einfachen Negation) zunehmend die Oberhand; zum Beispiel:

\footnotetext{
${ }^{32}$ Tsches geht auf ungarisch csösz ('Feldhüter') zurück.

${ }^{33}$ Die Erforschung der doppelten Negation besitzt ferner erhebliche sprachtheoretische Relevanz, da sie u.a. eine Herausforderung der FREGE'schen kompositionellen Semantik darstellt (vgl. FREGE 1994).
} 
(58) Jetz: tuar i: niks it:. (SD: Jetzt tue ich nichts nicht. - Wohl in Anlehnung an ungar. ,semmit sem“ [= nichts nicht]).

(59) Di:a Haut ka: ma wedr brá ta wedr niks. (SD: Diese Haut kann man weder braten weder nichts [noch für etwas anderes verwenden]).

Das Auftreten von doppelter Negation im Deutschen könnte andererseits womöglich auch als dialektologisch-sprachhistorisches Relikt gedeutet werden. In diesem Fall hat aber wohl der Einfluss des Ungarischen dieses Phänomen gestützt, sodass die obigen Befunde kontaktlinguistisch auf jeden Fall relevant sind.

Bei diesen Erscheinungen liegen (noch) die älteren Strukturen und (schon) die jüngeren Formen des ungarndeutschen Dialekts parallel vor, was den kontinuierlichen Sprachinnovationsprozess eindrücklich dokumentiert. So kommt in den in diesem Punkt besprochenen Belegen wieder einmal der Prozesscharakter bzw. die Dynamik von Sprachenkontakterscheinungen überzeugend zum Ausdruck.

3.9. Ein wohl schon als ,syntaktisch-pragmatisch“ einzustufender Transfer lässt sich im folgenden Fall dokumentieren:

(60) I: ha:n em Kohl Kanzlar gschrieba, abri: wä: eiß it:, kriag i: a Antwort odr it:. (SD: Ich habe dem Kohl Kanzler geschrieben, aber ich weiß nicht, kriege ich eine Antwort [= ob ich eine Antwort kriege] oder nicht.)

Im Ungarischen stehen - bis auf den „Dr.“, der auch vor dem Namen geführt werden kann sämtliche Titel, Ränge, Amtsbezeichnungen etc. hinter dem Familiennamen. So kann vermutet werden, dass dieser syntaktisch-pragmatische Sprachusus bei der Entstehung des Satzes Nr. (60) Pate gestanden hat.

3.10. Das nächste Beispiel demonstriert, dass Hybridität im untersuchten Material auch anhand des Transfers von grammatischen Beziehungen und Funktionen zur Geltung kommt:

(61) Jetz: ga:ud jäscha äl: uf: Baja. (SD: Jetzt gehen sie ja schon alle auf [= nach] Baja.)

In diesem Satz wird die Sublativus-Relation (vgl. Fußnote 12) des Ungarischen mittels der deutschen Präposition auf ausgedrückt, ${ }^{34}$ was im ausgewerteten Material sehr häufig vorkommt.

Besonders geläufig sind Transferenzen im Sinne einer Lehnbildung von Verbrektionen, Kollokationen und Verknüpfungsregulierungen oder -konventionen, etwa:

(62) Uf d Komonischta team:a it: szavazni. (SD: Auf [= für] die Kommunisten tun wir nicht stimmen.)

Die Verwendung der deutschen Präposition auf erklärt sich vermutlich mit dem Vorbild des ungar. Sublativsuffixes -ra/-re, wegen der ungarischen Verbrektion valakire/valamire szavaz $=$ 'auf jmdn./etw. stimmen'.

Die in den Belegen Nr. (61) und (62) vorliegenden Besonderheiten treten z.B. auch in manchen (älteren) binnendeutschen Dialekten auf. Aber obwohl diese Erscheinungen als

\footnotetext{
${ }^{34}$ Allerdings ist in solchen operativen Funktionen die Verwendung der direktionalen Präposition auf statt $n a c h$ in (binnen)deutschen Dialekten nicht unbekannt.
} 
mundartliche Merkmale angesehen werden, ist dem deutsch-ungarischen Sprachenkontakt insofern eine Bedeutung zuzumessen, als er diese Besonderheiten stützt bzw. konserviert.

Ferner wird in der Funktion des standarddeutschen Indefinitpronomens $\operatorname{man},{ }^{35}$ wie dies beispielsweise auch im Beleg Nr. (32) der Fall war, wohl in Analogie zum Ungarischen) am häufigsten auf die finite Verbform in der dritten Person Plural zurückgegriffen.

3.11. Kontaktbeispiele, die auch in den Kompetenzbereich der Wortbildung gehören, wurden bereits oben angeführt. Dieser Thematik muss eine nicht unwichtige Rolle zukommen, ist doch das Deutsche eine ausgesprochene Wortbildungssprache, in der zur sprachlichen Erfassung der Welt in sehr starkem Maße Wortbildungsmittel eingesetzt werden. Da die Wortbildungslehre ein überaus komplexes und transdisziplinäres Gebiet darstellt, wird sie - ungeachtet ihres mittlerweile erheblichen wissenschaftlichen Kenntnisstandes - in den Fachpublikationen nach wie vor different behandelt. SCHWING (1993: $166 \mathrm{ff}$.) etwa positioniert sie in der Flexionsmorphologie, MATTHEWs (2002: 37) versteht sie als Teil der „lexical morphology“ und TYROLLER (2003: 183 ff.) handelt sie im Rahmen der Grammatik ab. SIEBERT (1999) geht der Frage nach der Verortung der Wortbildung ebenfalls innerhalb der Grammatik nach. Andere Linguisten, wie EICHINGER, vertreten den Standpunkt, dass sich die Wortbildung nicht mehr mit den Mitteln der Morphologie darstellen lässt und die Wortbildungslehre daher als ,ein Bereich der Linguistik [zu] verstehen [ist], der eine eigenständige Stellung in einem Grenzraum zwischen Flexionsmorphologie, Syntax und Lexikon hat" (2000: 176). Diesen Sonderstatus der Wortbildung thematisieren auch BARZ (2000: 300), NAUMANN (2000: 1) und DoNALIES (2005: 14).

Angesichts der Tatsache, dass die Wortbildung also zweifellos viele Bereiche der Sprache tangiert, soll sie im vorliegenden Aufsatz nicht gesondert, sondern im Rahmen der Grammatik thematisiert werden. Über die bisher angeführten Befunde hinaus sind im untersuchten Korpus hinsichtlich der Wortbildung folgende weitere Aspekte von Belang.

Die Belege zeigen mehrere unterschiedliche Phänomene. Beispielsweise findet gelegentlich nicht nur eine hybride, sondern eine „duale Wortbildung“ statt (ein Beispiel dafür ist etwa Nr. 4), d.h. es wird zugleich auf die entsprechenden Wortbildungssuffixe beider Sprachen zurückgegriffen, so wie in: Owoda:schr ('Kindergärtler' < ungar. óvoda 'Kindergarten' sowie das ungarische Nominalsuffix $-\boldsymbol{s}+$ das deutsche Suffix -er); bisweilen können solchen hybriden Bildungen weitere Derivationsmorpheme angehängt werden, vgl. das Diminutivum Owoda:schrla. Dieser Beleg weist andererseits nach, dass für die Substantivderivation auch komplexe Konstituenten in Frage kommen, d.h. solche, die selbst wiederum zusammengesetzt sind.

Aus vielen Belegen geht hervor, dass lexikalisch-semantische Transferenzen aus dem Ungarischen regulär den Wortbildungsmechanismen des deutschen Basisdialekts unterworfen werden, etwa beim Diminutiv, z.B. vers ('Gedicht') $\rightarrow$ Versle (Basis ungarischer Provenienz + schwäbisch-alemannisches Diminutivsuffix -le):

(63) Nu so a kle:is Versle. (SD: Nur so ein kleines Gedichtlein.)

\footnotetext{
${ }^{35}$ Das als ,nominativisch gebrauchte Sammelbezeichnung, oft statt des bestimmteren unsereiner, wir oder sie“ (ERBEN 1980: 218) benutzt wird.
} 
sowie bei Kose- bzw. Spottnamen, z.B. dr Bácsile ('Onkelchen') aus bácsi + Diminutivsuffix -le. Bei diesen Belegen für affixale Modifikationen wurden für die Substantivderivation - im Gegensatz zum obigen Owoda:schr-Beispiel - simplizische Konstituenten verwendet.

SCHWOB (1971: 56 f.) etwa hat anhand seiner Betrachtungen an sathmarschwäbischem Sprachstoff konstatiert, dass sich Belege für ,solche Mischbildungen“ am leichtesten „im Bereich der Verkleinerungssilben“ finden lassen, nach dem Strukturtyp: „ungarisches Fremdwort mit schwäb. Diminutivsuffix“ (1971: 57). Derartige Fälle habe ich oben aus Hajosch expliziert. Mein Material geht aber deutlich über diesen sprachlichen Datentyp hinaus, wie das auch die nachfolgenden Ausführungen zeigen. So wird etwa bei der Movierung maskuliner Berufsbezeichnungen den ungarischen Transferaten meist das entsprechende Feminin-Bildungs-Suffix des deutschen Grunddialekts - $e$ angefügt, was in etwa dem standarddeutschen -in entspricht. Solche hybriden Formen sind z.B. $d$ Fönök:e ('Chefin' < ungar. fönök 'Chef') oder d Színésze ('Schauspielerin' < ungar. színész 'Schauspieler'):

(64) Iahra Muat:r ischt a színésze. (SD: Ihre Mutter ist eine Schauspielerin.)

Diese Suffigierung kann auch bei bereits hybriden Bildungen erfolgen, so entsteht z.B. $d$ Fölvidékre ('Oberländerin') aus Fölvidékr (der ungar. Landschaftsname Fölvidék + das dt. Derivationssuffix -er) < ungar. fölvidéki ('Oberländer').

Manche Belege zeigen den umgekehrten Fall, indem deutsche Lexeme mit ungarischen Wortbildungsaffixen versehen werden. Mitunter werden an einen deutschen Stamm sogar mehrere Suffixe des Ungarischen angehängt, z.B. Ni:nike ('Opi'). Ni:ne bedeutet 'Opa', die ungarisch beeinflusste Koseform lautet $N i: n i{ }^{36}$ Hinzu tritt das ungarische Kosenamenbildungssuffix $-\boldsymbol{k} \boldsymbol{a}$.

Es stehen also für die Wortbildungs-Hybridität beide „Richtungen“ offen. Deutsche Suffixe werden ungarischen Transferaten nicht nur zur Bildung grammatischer Formen und Relationen angehängt, sondern auch zur Konstituierung neuer Wörter. Eine gleichsam spektakuläre Wortbildung geht im untersuchten ungarndeutschen Dialekt etwa aufgrund des derben ungarischen ${ }^{37}$ Lexems picsa ('Fotze', 'Arsch') vor sich: Das entstandene Substantiv $d$ Bitschka findet in der Bedeutung 'schlechte Frau' Verwendung, aus dem dann - mit einer leichten semantischen Verschiebung - das Adjektiv pitschkesch, z.B. pitschkischi Hosa 'anzüglich anstößige Hose(n)' gebildet werden kann.

In der kommunikativen Praxis tritt nicht nur das Phänomen auf, dass Elemente ungarischer Provenienz unter Zuhilfenahme von deutschen Affixen und nach den Regeln des deutschen Dialekts an Wortbildungsmechanismen beteiligt sind. Es gibt auch Erscheinungen, bei denen aus deutschen Spracheinheiten nach den Mustern der ungarischen Wortbildung bestimmte Wortbildungsprodukte entstehen. Es hat sich nämlich gezeigt, dass auch ungarische Wortbildungsmodelle in den ungarndeutschen Dialekt übernommen werden können. Dadurch ergeben sich des Öfteren Formen, die aus binnendeutscher Perspektive vielleicht als befremdlich oder unverständlich erscheinen, wie die Verbalisierung von Substantiven. Vgl.

\footnotetext{
${ }^{36}$ Das ungarische Kosenamenbildungssuffix $-\boldsymbol{i}$ hat die ungewöhnliche Eigenschaft, dass es in der Regel „,nicht an das Wort, sondern an dessen in irgendeiner Weise verstümmelte Variante tritt“ (vgl. KIEFER 1999: 226).

${ }^{37}$ Eigentlich ist es - dem ,Etymologischen Wörterbuch des Ungarischen“ zufolge - ein ,süd- oder westslawisches Lehnwort“" (BENKÖ 1993: 1154).
} 
$k a: t^{38}$ (,karteln“) (= 'Karten spielen') < wohl nach dem Muster des gleichartigen ungarischen adnominalen Verbs kártyázik ['ka:rtja:zik]:

(65) En dr Kän klstuba ha: ma oft ka:ted. (SD: In der Spinnstube haben wir oft gekartelt [= Karten gespielt].)

Oder: kafe:a ('Kaffee trinken'< ungar. kávézik), im Perfekt: h. kafe:|ed. Hin und wieder entstehen sogar Homonyme zu Verben der standarddeutschen Varietät, z.B. kriaga ('Krieg führen'< ungar. háborúzik) vs. kriegen ('bekommen'). Solche adnominalen Verben werden auch aus dem Ungarischen transferiert und mit dem Verbsuffix des deutschen Dialekts versehen, wodurch das Ausdruckspotenzial erheblich bereichert wird, zumal die deutsche Standardvarietät solche Verben nicht kennt. Vgl.:

(66) Am liabschta ga:ud sie halt he:it die diszkózna. (SD: Am liebsten gehen sie halt heute zu „diskotheken“ [aus dem Substantiv diszkó 'Disco' gebildetes Verb (< ungar. diszkózik), d.h. sich in der Diskothek amüsieren].)

Belege gibt es auch im Hinblick auf andere Wortarten, z.B. bei Adjektiven: muat:resch ('Mutterkind, das Kind hängt sehr an der Mutter' < ungar. anyás). Recht oft ist dieser Kontakteinfluss bei „Fremdwörtern“ wirksam, z.B. Tscheslowa:kien 'Tschechoslowakei’ (< nach ungar. Csehszlovákia).

Man kann unschwer erkennen, dass die untersuchte Kontaktvarietät auch wortbildungsmäßig manche innovative und kreative Phänomene aufweist. Als modernes Beispiel ist auch das Lexempaar Ni:ni-Sit:er bzw. Na:na-Sit:er anzusehen. Nach dem Vorbild des (eigentlich englischen) Kompositums Babysitter, das in Hajosch quasi als ungarisches Wort wahrgenommen wird, nennt man neuerdings Personen, die ältere kranke oder behinderte Männer betreuen Ni:ni-Sit:er (Ni:ni = 'Opa'), während die Pfleger älterer Frauen heute Na:na-Sit:er heißen (Na:na = 'Oma').

\section{4. $\quad$ Fazit und Perspektiven}

Aus der obigen „Sprachdegustation“ geht hervor, dass sich anhand der Verwendung von grammatischen Formen, Strukturen und Mustern in einer spezifischen 'Kultur von Mehrsprachigkeit' - in einem dichten sprachkommunikativen Beziehungsgeflecht zwischen mehreren Sprach(varietät)en und kulturellen Systemen - zahlreiche und mannigfaltige Realisierungsklassen und -typen von kommunikativem Synkretismus und sprachlicher Hybridität auf verschiedenen Ebenen herausarbeiten ließen. ${ }^{39}$ Bei dieser grammatischen Zweisamkeit von Deutsch und Ungarisch sei betont, dass die in Frage stehende Kontaktvarietät kein „Mixtum compositum“ ist, d.h. nicht als bloße Summe von $\mathrm{L}_{1}$ und $\mathrm{L}_{2}$ (bzw. L3 usw.) anzusehen ist, sondern auch weitere, qualitativ neue Möglichkeiten offeriert (vgl. SJÖLIN 1976). Es geht also im Sinne von BECHERT/WILDGEN (1991: 3) auch nicht um ein simples Modell mechanischer Mischung, bei der sich das Redeprodukt restlos in Bestandteile der einen oder anderen Varietät zerlegen lässt und bei der erwartet wird, dass diese Bestandteile klar erkennbar und den Herkunftsvarietäten ohne Weiteres zuzuordnen

\footnotetext{
${ }^{38}$ Dieses „Hajoscher“ Verb ist auch im Transkript von KÜNZIG/WERNER (1969: 16) belegt.

${ }^{39}$ Da für die Ungarndeutschen ein kontextgebundener bilingual-oszillierender Sprech- bzw. Gesprächsstil charakteristisch ist (vgl. FöLDES 2005: 264), wäre es wohl vorerst kaum möglich zu modellieren, (soziologisch betrachtet) wer, welche - hybride oder ungemischte - grammatische Form in welchen Situationen sowie zu welchen kommunikativen Zwecken verwendet und durch welche komplexe Gebrauchssystematik sich die beobachteten Transferenzphänomene auszeichnen.
} 
sind. Vielmehr konstituieren sich dabei oft, wie etwa die Belege Nr. 43-46 und 50 nahe legen, weitgehend neue, „Dritte-Raum-Qualitäten“ (zwischen den Kulturen), die etliche Differenzen und scheinbar Unvereinbares in eine Relation bringen sowie Grenzen zwischen „Innen“ (dem „Eigenen“) und „Außen“ (dem „Fremden“) verschieben bzw. verschwinden lassen, vgl. RUTHERFORD (1990: 207 ff.) und FÖLDES (2005: 68 ff.). Bei dieser neuen, synthetisierenden Sprach- bzw. Kommunikationskultur handelt es sich schließlich um eine ausgehandelte Vermischung kulturellen Verhaltens und sprachlicher Substanz zweierlei Abkunft. Im Zeichen einer Infragestellung jeglicher auf binären Differenzmodellen beruhenden Ordnung könnte man wohl auch - als möglicher Überbegriff für Konzepte kultureller Vermischungen und Überlagerungen - von einer ,Transdifferenz ${ }^{640}$ sprechen.

Anhand der beobachteten Großbaustelle 'Grammatik' stellt die Sprachenmischung sowohl bezüglich der Objekt- als auch der Metaebene ein überaus facettenreiches Problemfeld dar. Für den hier vorliegenden Fall bedeutet das: Phänomene der Hybridität funktionieren selbst in einer ultimativen Sprachenkontakt-Situation nicht ganz ohne System und Gesetzmäßigkeiten, ${ }^{41}$ sowohl linguistisch als auch sozial und kulturell. Man muss folglich davon ausgehen, dass Elemente, Strukturen und Modelle des Ungarischen im Sinne einer „geordneten Selektion“ (HASSELMO 1972: 261 ff.) in die ungarndeutsche Basisvarietät eingebettet wurden und werden bzw. an den Umschaltungsprozeduren des Kodes beteiligt sind. Damit soll den Aussagen derjenigen Forscher ausdrücklich widersprochen werden, die in solchen Konstellationen eine „regellose“ und „durch nichts motivierte Sprachmischung“ (z.B. BRAUN 1937: 125, STOLT 1964: 13) oder etwas völlig Unsystematisches sehen, vgl. z.B. auch jüngere Publikationen von BOUTERWEK (1990: 21) und GLONING (1994: 17). Die kontakt-induzierten Systeme und Konstruktionen des ungarndeutschen Basisdialektes haben sich als funktionstüchtig erwiesen und es tauchen im Fahrwasser der Syntax kaum Turbulenzen auf. Bezogen auf die mentale Repräsentation ergibt sich, dass es sich wohl - im Gegensatz zum Lexikon - um zwei voneinander getrennte syntaktische Systeme handelt. ${ }^{42}$ Zur allgegenwärtigen Umgebungs- bzw. Referenzsprache Ungarisch bestehen immer mehr strukturelle Affinitäten. Sie liefert den strukturbildenden Hintergrund, das ständig präsente Modell, welches das intuitive grammatische Verständnis beim Dialektsprecher steuert.

Vielfach laufen Sprachproduktion und kommunikatives Verhalten über Transferstrategien ab. Die morphosyntaktischen Neuerungen (vgl. die ugyan-Komposita; siehe Belege 43, 44 etc.) gehen auf die modellbildende Wirkung des Ungarischen zurück, ${ }^{43}$ Einbußen an grammatischer Funktionalität sind dabei keine notwendige Folge. Das wird nicht zuletzt daran deutlich, dass nicht ausschließlich rezipierende Transferenzen etc. vorliegen, sondern dass der behandelte ungarndeutsche Dialekt eine nicht zu unterschätzende Eigendynamik und eine Art „Kontaktkreativität“ entfaltet (vgl. FöLDES 2005: 252 ff.). ${ }^{44}$

\footnotetext{
${ }^{40} \mathrm{Zu}$ diesem aus der Erlanger Kulturhermeneutik stammenden ,concept in progress“ vgl. ALLOLIONÄCKE/KALSCHEUER/MANZESCHKE (2005).

${ }^{41}$ Zur Problematik und Abgrenzung von „Prinzipien“, „Regeln“ und „Gesetzmäßigkeiten“ in der Sprachwissenschaft vgl. z.B. WOLFF (2004: 33).

${ }^{42}$ Die Modellierung der Repräsentation des bi- bzw. multilingualen mentalen Lexikons ist äußerst kompliziert, die Vernetzung ist hier zweifellos komplexer als bei nur einer Sprache. Die aktuelle Literatur sieht eine wichtige Forschungsaufgabe z.B. in der Präzisierung dessen, unter welchen Bedingungen und für welche Teile des Lexikons die involvierten Sprachen beim Sprecher getrennt oder integriert gespeichert und verarbeitet werden und geht kaum mehr von einer Dichotomie (koordiniert vs. verschmolzen) aus, sondern von einem „multidimensionalen Kontinuum“ (vgl. MiLISTE 2002: 257).

${ }^{43}$ Durch die bilinguale Sprachkreativität der mehrsprachigen Sprecher wird hier die Motivation der ursprünglich ungarischen Komposita transparent.

${ }^{44}$ Z.B. bei den mit ak:a:r- gebildeten hybriden Pronominaladverbien; siehe Belege (38), (39) etc.
} 
Bei der untersuchten 'gelebten Hybridität' sind unter dem „Triumvirat“" von Ortsdialekt, Ungarisch und Standarddeutsch mitunter sogar Aspekte des Wechselgefüges von Sprache, Kultur und Kognition in Erscheinung getreten. Da die Hauptakteure des Kontaktgeschehens (ein deutscher Ortsdialekt und Ungarisch) sowohl genetisch als auch typologisch disparate Sprachen sind, bietet die durchgeführte Analyse (neben kontaktlinguistischen Ergebnissen) auch Aufschlüsse für Aspekte der Sprachtheorie und der Theorie bzw. der Methodologie von Inter- bzw. Transkulturalität. Meine Analyse verdeutlicht ferner, dass das besprochene „Kontaktdeutsch“ in vielfacher Hinsicht den „sprachkommunikativen Fingerabdruck“ des Ungarischen als dominante Kontakt- und Prestigesprache aufweist und mit seinen phänotypischen Merkmalen sowie Relationen einen spezifischen Varietätentyp darstellt.

Das Untersuchungsfeld 'Morphosyntax im Sprachen- und Kulturenkontakt' erfordert künftig noch weitere Detailforschungen über Natur, Ausmaß und Folgen evidenter und latenter Kontaktmechanismen. Wenn nämlich im Sog kontaktsprachlicher Muster z.B. ganze Paradigmenfelder transferiert werden, so hat man es gar mit einer Übernahme komplexer kognitiver Muster von einer Diskurs- bzw. Kodegemeinschaft in die andere zu tun, mit denen gleichzeitig die Übertragung bestimmter Sichtweisen und Erklärungsmodelle einhergeht (vgl. JANSEN 2002: 59, FöLDES 2005: 205). Die Erforschung dieser Komplexität verlangt ein wahrlich inter- bzw. transdisziplinäres Herangehen.

\section{Literatur}

ACKERMANN, Andreas (2004): Das Eigene und das Fremde: Hybridität, Vielfalt und Kulturtransfers. In: JAEGER, Friedrich/RÜSEN, Jörn (Hrsg.): Handbuch der Kulturwissenschaften. Band 3: Themen und Tendenzen. Stuttgart/Weimar: Metzler. S. 139-154.

Allolio-NÄCKe, Lars/Kalscheuer, Britta/ManZeSCHKe, Arne (Hrsg.) (2005): Differenzen anders denken. Bausteine zu einer Kulturtheorie der Transdifferenz. Frankfurt a.M./New York: Campus.

Bachtin, Michail M. (1979): Die Ästhetik des Wortes. Frankfurt a.M.: Suhrkamp. (Edition Suhrkamp; 967).

BARBA, Katharina (1982): Deutsche Dialekte in Rumänien. Die südfränkischen Mundarten der Banater deutschen Sprachinsel. Wiesbaden: Steiner. (ZDL, Beihefte; 35).

BARZ, Irmhild (2000): Zum heutigen Erkenntnisinteresse germanistischer Wortbildungsforschung. Ein exemplarischer Bericht. In: BARZ, Irmhild/SCHRÖDER, Marianne/FIX, Ulla (Hrsg.): Praxis- und Integrationsfelder der Wortbildungsforschung. Heidelberg: Winter. (Sprache - Literatur und Geschichte; 18). S. 299-316.

BÁTORI, István (1980): Russen und Finnougrier. Kontakt der Völker und Kontakt der Sprachen. Wiesbaden: Harrassowitz. (Veröffentlichungen der Societas Uralo-Altaica; 13).

BECHERT, Johannes/WILDGEN, Wolfgang [Unter Mitarbeit von Christoph SCHROEDER] (1991): Einführung in die Sprachkontaktforschung. Darmstadt: Wiss. Buchgesellschaft. (Die Sprachwissenschaft).

BENKŐ, Loránd (Hrsg.) (1993): Etymologisches Wörterbuch des Ungarischen. Budapest: Akadémiai.

Berend, Nina/Jedig, Hugo (1991): Deutsche Mundarten in der Sowjetunion. Geschichte der Forschung und Bibliographie. Marburg: Elwert. (Schriftenreihe der Kommission für Ostdeutsche Volkskunde in der Deutschen Gesellschaft für Volkskunde; 53).

BERNER, Ulrich (1982): Untersuchungen zur Verwendung des Synkretismus-Begriffes. Wiesbaden: Harrassowitz. (Göttinger Orientforschung: Grundlagen und Ergebnisse; 2).

BнавнA, Homi T. (2000): Die Verortung der Kultur. Tübingen: Stauffenburg. (Stauffenburg discussion 5).

BOUTERWEK, Friedrich (1990): Sprachkontakt und Dialektkontakt - zwei ungleiche Brüder? In: Verein d. Freunde d. im Mittelalter von Österreich aus besiedelten Sprachinseln/Komm. für Mundartkunde u. Namenforschung d. Österr. Akad. d. Wiss. (Hrsg.): Mundart und Name im Sprachkontakt. Festschrift für Maria Hornung zum 70. Geburtstag. Wien: VWGO. (Beiträge zur Sprachinselforschung; 8). S. 17-34. 
BRAUN, Maximilian (1937): Beobachtungen zur Frage der Mehrsprachigkeit. In: Göttingische Gelehrte Anzeigen 199. 4. S. 115-130.

CARstAirs-McCarthy, A[ndrew] (1994): Syncretism. In: AsHER, R. E./SiMPSON, J. M. Y. (Eds.): The Encyclopedia of Language and Linguistics. Vol. 8. Oxford/New York/Seoul/Tokyo: Pergamon Press. S. 4453-4454.

CLYNE, Michael (1992): Zur Gegenwart und Zukunft der deutschen Sprache in Australien. In: Muttersprache 102. 3. S. 193-203.

DIEWALD, Gabriele (1997): Grammatikalisierung. Eine Einführung in Sein und Werden grammatischer Formen. Tübingen: Niemeyer. (Germanistische Arbeitshefte; 36).

DONALIES, Elke (2005): Die Wortbildung des Deutschen. Ein Überblick. Zweite, überarb. Aufl. Tübingen: Narr. (Studien zur Deutschen Sprache; 27).

EICHINGER, Ludwig M. (2000): Deutsche Wortbildung. Eine Einführung. Tübingen: Narr. (Narr Studienbücher).

ERBEN, Johannes (1980): Deutsche Grammatik. Ein Abriß. 11., völlig neubearb. Aufl. München: Hueber.

FILIPOVIć, Rudolf (1986): Teorija jezika u kontaktu. Zagreb: Jugoslavenska Akademija Znanosti i Umjetnosti; 59, 1: Razred za Filološke Znanosti).

FLEISCHER, Wolfgang/BARZ, Irmhild [Unter Mitarbeit von Marianne SCHRÖDER] (1995): Wortbildung der deutschen Gegenwartssprache. 2., durchges. u. erg. Aufl. Tübingen: Niemeyer. (Studienbuch).

FöLDES, Csaba (1996): Mehrsprachigkeit, Sprachenkontakt und Sprachenmischung. Flensburg: Univ. (Flensburger Papiere zur Mehrsprachigkeit und Kulturenvielfalt im Unterricht; 14/15).

FÖLDES, Csaba (2003): Interkulturelle Linguistik: Vorüberlegungen zu Konzepten, Problemen und Desiderata. Veszprém: Universitätsverl./Wien: Praesens. (Studia Germanica Universitatis Vesprimiensis; Suppl.; 1).

FöLDES, Csaba (2005): Kontaktdeutsch: Zur Theorie eines Varietätentyps unter transkulturellen Bedingungen von Mehrsprachigkeit. Tübingen: Gunter Narr Verlag.

FreGE, Gottlob (1994): Funktion, Begriff, Bedeutung: Fünf logische Studien. Hrsg. und eingel. von Günther Patzig. 7., bibliogr. erg. Aufl. Göttingen: Vandenhoeck \& Ruprecht. (Kleine Vandenhoeck-Reihe; 1144).

GERESCHER, Konrad (o.J., wahrscheinlich 1993): Mundart-Sprüche aus Bereg/Bački Breg. Szeged: AK Mundart/Brauchtum, Deutsch-Ungarischer Freundeskreis.

GIRNTH, Heiko (2000): Untersuchungen zur Theorie der Grammatikalisierung am Beispiel des Westmitteldeutschen. Tübingen: Niemeyer. (Reihe Germanistische Linguistik; 223).

GLONING, Heike (1994): Sprachliche Interferenzen im donauschwäbischen Siedlungsraum und ihre Bewertung durch die Sprecher. In: GeHL, Hans/Purdela SutARU, Maria (Hrsg.): Interferenzen in den Sprachen und Dialekten Südosteuropas. Tübingen: Institut für donauschwäb. Geschichte und Landeskunde. (Materialien; 4/1994). S. 17-30.

GodDARD, Cliff/WIERZBICKA, Anna (2003): Sprache, Kultur und Bedeutung: Kulturvergleichende Semantik. In: PÖRINGS, Ralf/ScHMITZ, Ulrich (Hrsg.): Sprache und Sprachwissenschaft. Eine kognitiv orientierte Einführung. 2., überarb. u. akt. Aufl. Tübingen: Narr. (Narr Studienbücher). S. 139-162.

GRIMM, Jacob und Wilhelm (1860): Deutsches Wörterbuch. Band 2. Leipzig: Hirzel.

HASSELmo, Nils (1972): Code-Switching as Ordered Selection. In: FIRCHOW ScHERABON, Evelyn/GRIMSTAD, Kaaren/Hasselmo, Nils/O’NeIL, Wayne A. (Eds): Studies for Einar Haugen. Presented by Friends and Colleagues. The Hague/Paris: Mouton. (Janua Linguarum; Series Maior; 59). S. 261-280.

HofFMANN, Charlotte (1997): An Introduction to Bilingualism. 5. impr. London/New York: Longman. (Longman Linguistics Library).

Hopper, Paul J./TraugotT, Elizabeth Closs (2000): Grammaticalization. Repr. Cambridge: Cambride Univ. Press. (Cambridge Textbooks in Linguistics).

HUFEISEN, Britta (1995): Englisch bei deutschsprachigen Immigranten in Kanada. In: Muttersprache 105. 3. S. 243-251.

HUTTERER, Claus Jürgen (1993): Konvergenzen in der Volkskultur der Deutschen im Karpatenbecken. (Am Beispiel der Sprachentwicklung). In: BASSOLA, Péter/HESSKY, Regina/TARNÓI, László (Hrsg.): Im Zeichen 
der ungeteilten Philologie. Festschrift für Professor Dr. sc. Karl Mollay zum 80. Geburtstag. Budapest: ELTE. (Budapester Beiträge zur Germanistik; 24). S. 147-170.

IVANOV, V. V. (1990): Kontakty jazykovye. In: JARCEVA, V.N. (Glavn. red.): Lingvističeskij enciklopedičeskij slovar'. Moskva: Sovetskaja enciklopedija. S. 237-238.

JANSEN, Silke (2002): Metaphern im Sprachkontakt - anhand von Beispielen aus dem französischen und spanischen Internetwortschatz. In: metaphorik.de 03/2002. S. 44-74. Gesehen im Internet unter metaphorik.de am 19.03.2005).

KAllMEYeR, Werner/KeIM, Inken/Aslan, Sema/CInDARK, Ibrahim (2002): Variationsprofile. Zur Analyse der Variationspraxis bei den „Powergirls“. Gesehen im Internet unter: http://www.idsmannheim.de/prag/sprachvariation/fgvaria/Variationsprofile.pdf am 11.05.2005.

KENESEI, István/VAGO, Robert M./FenYVESI, Anna (1998): Hungarian. London/New York: Routledge. (Descriptive Grammars).

KERESZTES, László (1999): Praktische ungarische Grammatik. 3., verbess. Aufl. Debrecen: Debreceni Nyári Egyetem. (Hungarolingua).

KIEFER, Ferenc (1999): Alaktan. In: É. KISS, Katalin/KIEFER, Ferenc/SIPTÁR, Péter: Új magyar nyelvtan. 2. kiadás. Budapest: Osiris. S. 185-289.

KISS, Jenő (szerk.) (2001): Magyar dialektológia. Budapest: Osiris. (Osiris Tankönyvek).

KLASSEN, Heinrich (1994): Zur Sprachsituation in Siedlungsgebieten mit vorwiegend deutscher Bevölkerung in Rußland. In: WILD, Katharina (Hrsg.): Begegnung in Pécs/Fünfkirchen. Die Sprache der deutschsprachigen Minderheiten in Europa. Pécs: Univ. (Studien zur Germanistik; 2). S. 63-74.

KNAB, Elisabeth (1997): Wie die Nadwarer reda (Wie die Nadwarer reden). Mundart der Nadwarer. In: RICHTER, Georg: Geliebtes Nadwar. Erinnerungen an die verlorene Heimat H-6345 Nemesnádudvar. Horb am Neckar: Geiger-Verl. S. 189-194.

KNECHT, Tamás (1999): Lexikalische Interferenzen aus dem Rumänischen und Ungarischen in den schwäbischen Dialekten von Beschened und Petrifeld. In: GEHL, Hans/CiUBOTĂ, Viorel (Hrsg.): Interethnische Beziehungen im rumänisch-ungarisch-ukrainischen Kontaktraum vom 18. Jahrhundert bis zur Gegenwart. Satu Mare: Ed. Muzeului Sătmărean/Tübingen: Institut für donauschwäb. Geschichte und Landeskunde. S. 345-374.

KNECHT, Tamás (2001): Lexikalische Interferenzen in den sathmarschwäbischen Dialekten der Gemeinden Bescheneed, Petrifeld und Terem. In: GeHL, Hans (Hrsg.): Dialekt - Lehnwörter - Namen. Sprachliche Studien über die Sathmarer Schwaben. Tübingen: Institut für donauschwäb. Geschichte und Landeskunde. (Materialien; 12). S. 53-114.

KNIPF-KoMLÓSI, Elisabeth (2003): Die Substantivbildung in der Mundart. Ein Beitrag zur Substantivderivation am Beispiel einer ungarndeutschen Mundart. Budapest: ELTE. (Budapester Beiträge zur Germanistik; 42).

KÖNIG, Ekkehard/EisENBERG, Peter (1984): Zur Pragmatik von Konzessivsätzen. In: STICKEL, Gerhard (Hrsg.): Pragmatik in der Grammatik. Jahrbuch 1983 des Instituts für deutsche Sprache. Düsseldorf: Päd. Verl. Schwann-Bagel. (Sprache der Gegenwart; 60). S. 313-332.

KÜNZIG, Johannes/WERNER, Waltraut (1969): Die Rosibäs aus Hajós. Authentische Tonaufnahmen 1967 in Hajós. Freiburg i. Br.: Rombach. (Quellen deutscher Volkskunde; 2/Ungarndeutsche Märchenerzähler; 1).

LEUSCHNER, Torsten (2000): „,.., wo immer es mir begegnet, ...- wo es auch sei“. Zur Distribution von 'Irrelevanzpartikeln' in Nebensätzen mit w-auch/immer. In: Deutsche Sprache 28. S. 342-356.

LuHMANN, Niklas (2000): Soziale Systeme. Grundriß einer allgemeinen Theorie. Nachdr. Frankfurt a.M.: Suhrkamp. (Suhrkamp-Taschenbuch, Wiss.; 666).

MatThews, Peter H. (2002): Morphology. 2. ed. repr. Cambridge: Cambridge Univ. Press. (Cambridge Textbooks in Linguistics).

MELIKA, Georg (1994): Spracherhaltung und Sprachwechsel bei der deutschen Minderheit von Transkarpatien. In: BEREND, Nina/MATTHEIER, Klaus J. (Hrsg.): Sprachinselforschung. Eine Gedenkschrift für Hugo Jedig. Frankfurt a.M./Berlin/Bern/New York/Paris/Wien: Lang. S. 289-301. 
MiLISTE, Merje (2002): Modellierung von Mehrsprachigkeit im Hinblick auf den Erwerb von weiteren Fremdsprachen. In: Pavidis, Silvija/Taterka, Thomas (Hrsg.): Triangulum. Germanistisches Jahrbuch für Estland, Lettland und Litauen. Neunte Folge. Riga/Bonn: DAAD. (Reihe Germanistik). S. 253-266.

MÜLLER, Max (1965): Lectures on the Science of Language. Delivered at the Royal Institution of Great Britain in April, May \& June, 1861. Fifth Reprint. Delhi: Munshi Ram Manohar Lal.

NAUMANN, Bernd (2000): Einführung in die Wortbildungslehre des Deutschen. 3., neu bearb. Aufl. Tübingen: Niemeyer. (Germanistische Arbeitshefte; 4).

NAVRACSICS, Judit (1999): A kétnyelvü gyermek. Budapest: Corvina. (Egyetemi Könyvtár: Alkalmazott Nyelvészet).

RoELCKE, Thorsten (2002): Sprachtypologische Tendenzen der deutschen Gegenwartssprache. In: Leuvense Bijdragen 91. 3-4. S. 259-285.

RONNEBERGER-SIBOLD, Elke (1980): Sprachverwendung - Sprachsystem: Ökonomie und Wandel. Tübingen: Niemeyer. (Linguistische Arbeiten; 87).

RUTHERFORD, Jonathan (1990): The Third Space. Interview with Homi Bhabha. In: RuTHERFORD, Jonathan (Ed.): Identity, Community, Culture, Difference. London: Lawrence and Wishart. S. 207-221.

SAPIR, Edward (1921): Language. An Introduction to the Study of Speech. New York: Harcourt, Brace \& World. (A Harvest Book; HB7).

Schappelle, Benjamin Franklin (1917): The German Element in Brasil. Colonies and Dialect. Philadelphia: Americana Germanica Press. (Americana Germanica; 26).

SCHMIDT, Karl Horst (1998): Versuch einer geschichtlichen Sprachtypologie des Deutschen. In: BESCH, Werner/Betten, Anne/Reichmann, Oskar/SonderegGer, Stefan (Hrsg.): Sprachgeschichte. Ein Handbuch zur Geschichte der deutschen Sprache und ihrer Erforschung. 2., vollst. neu bearb. u. erw. Aufl. Berlin/New York: de Gruyter. (Handbücher zur Sprach- und Kommunikationswissenschaft; 2.1). S. 9931000 .

Schwalm, Paul (1979): Wörterbuch des Dialekts der Deutschen in Vaskút/Südungarn. A vaskúti németek tájszólásának szótára. Herausgegeben von FLACH, Paul. Neuenstein: Heim-Verl.

SCHWEDT, Herbert und Elke (1995): „Eine verfluchte Zeit“ - Aufzeichnungen von Maria L. Aus dem schwäbischen Dorf Hajós in Ungarn. In: Jahrbuch für deutsche und osteuropäische Volkskunde. Hrsg. im Auftr. der Kommission für Deutsche und Osteuropäische Volkskunde in der Deutschen Gesellschaft für Volkskunde. Bd. 38. Marburg: Elwert. S. 24-41.

ScHWING, Josef (1993): Grammatik der deutschen Mundart von Palotabozsok (Ungarn). Frankfurt a.M.: Hector. (Forum Phoneticum; 55).

SCHWOB, Anton (1971): Wege und Formen des Sprachausgleichs in neuzeitlichen ost- und südostdeutschen Sprachinseln. München: Oldenbourg. (Buchreihe der Südostdeutschen Historischen Kommission; 25).

SIEBERT, Susann (1999): Wortbildung und Grammatik. Syntaktische Restriktionen in der Struktur komplexer Wörter. Tübingen: Niemeyer. (Linguistische Arbeiten; 408).

SJÖLIN, B[o] (1976): Kodewechsel und Transferenz bei diglossischem Bilinguismus. Eine Typik der Voraussetzungen ihrer Entstehung. In: Studia Neophilologica 48. 2. S. 245-268.

STEPANOVA, N. N. (1983): K voprosu o sopostavitel'nom analize valentnych svojstv glagolov verchnenemeckich govorov Altaja. In: Edig, G. G. (otv. red.): Voprosy dialektologii nemeckogo jazyka. Respublikanskij sbornik nauCnych trudov. Omsk: Ped. Institut. S. 192-199.

StOLT, Birgit (1964): Die Sprachmischung in Luthers Tischreden. Studien zum Problem der Zweisprachigkeit. Uppsala: Almqvist \& Wiksells. (Stockholms Universitet: [Acta Universitatis Stockholmiensis/Stockholmer germanistische Forschungen]; 4).

TEKINAY, Alev (1987): Deutsche Einflüsse im Türkischen von Arbeitsmigranten. In: TeKINAY, Alev: Sprachvergleich Deutsch-Türkisch. Möglichkeiten und Grenzen einer kontrastiven Analyse. Wiesbaden: Reichert. S. 96-103.

TOMPA, József (1972): Kleine ungarische Grammatik. Leipzig: Verl. Enzyklopädie.

TYROLLER, Hans (2003): Grammatische Beschreibung des Zimbrischen in Lusern. Stuttgart: Steiner. (ZDL, Beihefte; 111). 
WILD, Katharina (1994): Statistisch-syntaktische Untersuchungen ungarndeutscher Mundarten. In: SCHWOB, Anton/FASSEL, Horst (Hrsg.): Deutsche Sprache und Literatur in Südosteuropa - Archivierung und Dokumentation. Beiträge der Tübinger Fachtagung vom 25.-27. Juni 1992. München: Verl.

Südostdeutsches Kulturwerk. (Veröff. des Südostdt. Kulturwerks, Reihe B, Wiss. Arbeiten; 66). S. 94-98.

WINFORD, Donald (2003): An Introduction to Contact Linguistics. Malden/Oxford/Melbourne/Berlin: Blackwell Publ. (Language in Society; 33).

WOLFF, Gerhart (2004): Deutsche Sprachgeschichte von den Anfängen bis zur Gegenwart. Ein Studienbuch. 5., überarb. u. aktual. Aufl. Tübingen/Basel: Francke. (UTB; 1581).

WURZEL, Wolfgang Ulrich (2001): Ökonomie. In: HASPELMATH, Martin/KÖNIG, Ekkehard/OSTERREICHER, Wulf/RAIBLE, Wolfgang (Eds.): Language Typology and Language Universals. Vol. 1. Berlin/New York: de Gruyter. (Handbücher zur Sprach- und Kommunikationswissenschaft; 20.1). S. 384-400.

ZIEGLER, Arne (1996): Deutsche Sprache in Brasilien. Untersuchungen zum Sprachwandel und zum Sprachgebrauch der deutschstämmigen Brasilianer in Rio Grande do Sul. Essen: Verl. Die Blaue Eule. (Kultur der Deutschen im Ausland; 2).

\section{Der Verfasser:}

Prof. Dr. Csaba Földes

Pannonische Universität Veszprém

Germanistisches Institut

Lehrstuhl für germanistische Linguistik

Füredi u. 2, Pf. 158

H-8201 Veszprém (Ungarn)

E-Mail: foldes@almos.vein.hu

Internet: www.vein.hu/german/ 